\title{
Determining optimal mulching, planting density, and nitrogen application to increase maize grain yield and nitrogen translocation efficiency in Northwest China
}

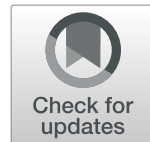

Xiukang Wang ${ }^{1 *}$ (D), Ge Wang ${ }^{1}$, Neil C. Turner ${ }^{2}$, Yingying Xing ${ }^{1 *}$, Meitian Li $^{1}$ and Tao Guo ${ }^{1}$

\begin{abstract}
Background: The combination of mulch with $N$ fertilizer application is a common agronomic technique used in the production of rainfed maize (Zea mays L.) to achieve higher yields under conditions of optimum planting density and adequate $\mathrm{N}$ supply. However, the combined effects of mulch, planting density, and $\mathrm{N}$ fertilizer application rate on plant $\mathrm{N}$ uptake and $\mathrm{N}$ translocation efficiency are not known. The objective of this study was to quantify the interaction effect of mulch, planting density, and $\mathrm{N}$ fertilizer application rate on maize grain yield, $\mathrm{N}$ uptake, $\mathrm{N}$ translocation, and $\mathrm{N}$ translocation efficiency. The experiment was arranged in a randomized complete block design with three factors ( 2 mulch levels $\times 2$ planting densities $\times 4 \mathrm{~N}$ fertilizer application rates) replicated four times.
\end{abstract}

Results: There was a significant interaction among mulch, plant density, and $\mathrm{N}$ fertilizer on maize grain yield, kernel number per cob, $\mathrm{N}$ uptake, $\mathrm{N}$ translocation, and $\mathrm{N}$ translocation efficiency. Averaged over the 3 years of the study, total plant $\mathrm{N}$ uptake at silking ranged from 79 to $149 \mathrm{~kg} \mathrm{~N} \mathrm{ha}^{-1}$ with no mulch and from 76 to $178 \mathrm{~kg} \mathrm{~N} \mathrm{ha}^{-1}$ with mulch. The $\mathrm{N}$ uptake at silking in different plant organs ranked as leaf $>$ grain $>$ stem $>$ cob. Averaged across all factors, the highest $\mathrm{N}$ translocation was observed in leaves, which was 59.4 and $88.7 \%$ higher than observed in stems and ears, respectively. The mean vegetative organ $N$ translocation efficiency averaged over mulch, planting density, and $\mathrm{N}$ fertilizer application rate treatments decreased in the order of leaf $>$ stem $>$ cob.

Conclusions: Mulch, planting density, and $\mathrm{N}$ fertilizer application rate not only have significant effects on improving maize grain yield and NUE, but also on N uptake, $\mathrm{N}$ translocation, and $\mathrm{N}$ translocation efficiency. Our results showed clearly that under high planting density, the combination of mulch and moderate $\mathrm{N}$ fertilizer application rate was the optimal strategy for increasing maize grain yield and $\mathrm{N}$ use efficiency.

Keywords: Dry matter accumulation, Nitrogen apparent recovery efficiency, Nitrogen assimilation amount, Nitrogen use efficiency, Nitrogen uptake

\footnotetext{
* Correspondence: wangxiukang@126.com; xingyingying610624@163.com ${ }^{1}$ College of Life Sciences, Yan'an University, Yan'an 716000, Shaanxi, China Full list of author information is available at the end of the article
}

(c) The Author(s). 2020 Open Access This article is licensed under a Creative Commons Attribution 4.0 International License, which permits use, sharing, adaptation, distribution and reproduction in any medium or format, as long as you give appropriate credit to the original author(s) and the source, provide a link to the Creative Commons licence, and indicate if changes were made. The images or other third party material in this article are included in the article's Creative Commons licence, unless indicated otherwise in a credit line to the material. If material is not included in the article's Creative Commons licence and your intended use is not permitted by statutory regulation or exceeds the permitted use, you will need to obtain permission directly from the copyright holder. To view a copy of this licence, visit http://creativecommons.org/licenses/by/4.0/. The Creative Commons Public Domain Dedication waiver (http://creativecommons.org/publicdomain/zero/1.0/) applies to the data made available in this article, unless otherwise stated in a credit line to the data. 


\section{Background}

Two-thirds of the food consumed by the world population is provided by maize, wheat (Triticum aestivum L.), and rice (Oryza sativa L.), and these three-plant species make up the clear majority of calories in human diets [1]. Maize is one of the most important cereal crops in Northwest China [2-4]. In the future, maize production will need to continue to grow to keep pace with population growth and future dietary changes $[5,6]$.

Increasing maize production is highly dependent upon chemical fertilizer inputs, especially nitrogen $(\mathrm{N})$ fertilizer [7]. Consequently, farmers often applied excessive amounts of $\mathrm{N}$ fertilizer to ensure high grain yields. This can result in huge $\mathrm{N}$ losses, low nitrogen use efficiency (NUE), high production costs, and environmental pollution [8-10]. The overuse of $\mathrm{N}$ fertilizer continues in semiarid regions of China and the rate of $\mathrm{N}$ fertilizer applied by small farmers is more than $360 \mathrm{~kg} \mathrm{~N} \mathrm{ha}^{-1}$ [11] in spite of the fact that the optimal $\mathrm{N}$ fertilizer application amount for maize has been shown to be only $180 \mathrm{~kg} \mathrm{~N} \mathrm{ha}^{-1}$ [1214 . Because of the serious negative effects caused by excessive fertilization, more and more attention has been given to determining optimal fertilization amounts and application methods [15-17]. Therefore, there is an urgent need to explore optimal field management options to improve grain yield and NUE, and to inform farmers about these options.

The combination of plastic film mulch and reasonable amounts of $\mathrm{N}$ fertilizer application can significantly increase maize grain yield in Northwest China [13]. Plastic mulch is widely used in global agricultural production. Plastic mulch is used on $20 \%$ of the cultivated land area in China [18]. Plastic mulch has been widely used in maize production in Northwest China, and can improve the topsoil temperature and conserve soil moisture [19]. Soil nitrate- $\mathrm{N}$ content is a useful indicator that helps to achieve economical yield in agricultural production [20]. The application of mulch and $\mathrm{N}$ fertilizer has a great impact on soil nitrate- $\mathrm{N}$ content and thus on maize growth and yield [11, 21-23]. Compared with no mulching, film mulching significantly increased yield and NUE (unit $\mathrm{N}$ yield) by up to $60 \%$ [24]. Film mulching is an effective way to improve NUE and crop yield by enhancing the interactions between $\mathrm{N}$ fertilizer application rate, water conservation, and yield [25].

Determining reasonable and optimum planting density is an effective measure to pursue in order to achieve high yield and high NUE. The main reasons for this are that 1) an optimum population canopy structure is closely related to proper plant density [26], and 2) the full yield potential supported by existing soil fertility and precipitation is obtained with optimal planting density [27]. Planting density has proven to be a very effective agronomic strategy to improve maize grain yield [28].
The optimum planting density of commercial maize in North America is about 75,000-80,000 seeds ha ${ }^{-1}$ plants under good agronomic conditions (i.e., no water or $\mathrm{N}$ stress), and most commercial maize breeding programs use a higher planting density (>160,000 seeds ha ${ }^{-1}$ ) to evaluate germplasm [29]. By contrast, the optimum maize planting density ranges from 49,850 to 65,180 seeds $\mathrm{ha}^{-1}$ in China $[26,30]$. Two years of field experiments showed that the highest maize grain yield was obtained when planting density was 90,000 seeds ha ${ }^{-1}$ [31]. Planting density management is an important agronomic method to influence maize yields [32]. The effect of planting density on maize yield and NUE was highly correlated with plant $\mathrm{N}$ uptake [33]. Thus, it is necessary to optimize planting density to meet high plant $\mathrm{N}$ uptake.

Maize plant $\mathrm{N}$ uptake was mainly affected by $\mathrm{N}$ uptake in grain [34]. Nitrogen in maize grain has two sources: uptake from roots and transport from vegetative organs [35]. Before physiological maturity, the absorbed N cannot be transported directly to the grain, but instead is stored in vegetative organs and then transported to the grain [36, 37]. As maize approaches physiological maturity, $\mathrm{N}$ uptake occurs simultaneously through direct transport from the soil to the grain through root uptake and translocation of stored $\mathrm{N}$ from vegetative organs to grain. Leaves and stems are the main sources of grain $\mathrm{N}$ [38]. Although a large number of agriculturalists focus on grain yield and NUE, there is a lack of information regarding how the three factors of mulch, planting density, and $\mathrm{N}$ fertilizer application rate change $\mathrm{N}$ translocation amount and $\mathrm{N}$ translocation efficiency from different maize vegetative organs.

The objective of this study was to determine how plastic mulch used in conjunction with high plant population density and $\mathrm{N}$ fertilizer application rate can increase maize grain yield, plant $\mathrm{N}$ uptake, $\mathrm{N}$ translocation, and $\mathrm{N}$ translocation efficiency. We hypothesized that $\mathrm{N}$ translocation to grain may be significantly increased by use of plastic mulch, increased planting density, and increased $\mathrm{N}$ fertilizer application rate. Exploring this hypothesis can provide guidance for farmers to adopt optimal management measures and also can provide a theoretical basis for improving yield and NUE.

\section{Results}

Aboveground dry matter accumulation, grain yield, and yield components

The individual factors of mulch, planting density, and $\mathrm{N}$ fertilizer application rate significantly $(p<0.001)$ affected aboveground DMA at silking and physiological maturity, and there was a significant two-way interaction between mulch and nitrogen fertilizer application rate and a three-way interaction among the factors at silking (Table 1). The differences in DMA between different $\mathrm{N}$ 
Table 1 Treatment effects ( $p$ values) for dry matter accumulation (DMA), and grain yield (GY) at silking (SS) and physiological maturity (PMS), using year, mulch, nitrogen fertilizer application rates, and planting density as four fixed factors

\begin{tabular}{llll}
\hline Sources & DMA-SS & DMA-PMS & GY \\
\hline Year $(Y)$ & $<0.001$ & 0.001 & $<0.001$ \\
Mulch (M) & $<0.001$ & $<0.001$ & $<0.001$ \\
Nitrogen ( $N)$ & $<0.001$ & $<0.001$ & $<0.001$ \\
Density (D) & $<0.001$ & $<0.001$ & $<0.001$ \\
$Y * M$ & ns & ns & ns \\
$Y * N$ & ns & ns & $<0.001$ \\
$Y * D$ & ns & ns & 0.006 \\
$M{ }^{*} N$ & $<0.001$ & $<0.001$ & $<0.001$ \\
$M{ }^{*} D$ & ns & 0.017 & $<0.001$ \\
$N{ }^{*} D$ & 0.001 & ns & $<0.001$ \\
$Y * M * N$ & ns & ns & ns \\
$Y * M * D$ & ns & ns & ns \\
$Y * N{ }^{*} D$ & ns & ns & ns \\
$M * N * D$ & 0.005 & ns & $<0.001$ \\
$Y * M * N * D$ & ns & ns & ns \\
\hline
\end{tabular}

Note: $n s$ Not significant $(p>0.05)$

fertilizer application rates was greater at physiological maturity than at silking stage (Figs. 1, 2). Mulching and high planting density increased maize DMA. Averaged across all $\mathrm{N}$ fertilizer application rates, mulch increased DMA by $6.7,11.8$, and $9.2 \%$ at physiological maturity under low planting density in 2016, 2017, and 2018, respectively, and increased DMA by $3.5,5.3$, and $4.8 \%$ under high planting density (Fig. 2). Averaged across all $\mathrm{N}$ fertilizer rates, DMA under high planting density was higher than DMA under low planting density with and without mulch in all 3 years (Figs. 1,2).

The individual factors of mulch, planting density, and $\mathrm{N}$ fertilizer application rate each had a significant influence on grain yield, and there was a significant interaction between each pair of factors. There was also a significant three-way interaction effect among these factors (Table 1). Increasing the $\mathrm{N}$ fertilizer application rate significantly increased grain yield (Fig. 3) under all mulch and planting density treatments, similar to what was observed for dry matter accumulation. Averaged across all $\mathrm{N}$ fertilizer application rates, plastic mulch increased grain yield by $4.2,6.3$, and $5.9 \%$ under the low planting density in 2016, 2017, and 2018, respectively, and increased grain yield by $20.2,21.4$, and $20.9 \%$ under high planting density (Fig. 3). Compared with low planting density, high planting density increased grain yield by $3.5,4.4$, and $5.7 \%$ under the no mulch treatment and increased grain yield by $18.1,27.2$, and $26.4 \%$ under the mulch treatment in 2016, 2017, and 2018, respectively
(Fig. 3). Averaged over all 3 years, the grain yield for $\mathrm{N}_{360}\left(11,490 \mathrm{~kg} \mathrm{ha}^{-1}\right)$ was the highest, which was 4.8 , 19.7 , and $111 \%$ higher than grain yield for $\mathrm{N}_{240}, \mathrm{~N}_{120}$, and $\mathrm{N}_{0}$, respectively, with the mulch and high planting density treatments (Fig. 3).

The individual factors of mulch, planting density, and $\mathrm{N}$ fertilizer application rate significantly affected all yield components (Table 2) except that mulch had no significant effect on cob number. The interaction among mulch, planting density, and $\mathrm{N}$ fertilizer application rate had a significant effect only on kernel number per cob. Averaged across $\mathrm{N}$ fertilizer application rate and mulch, cob length under low planting density was 3.1, 3.6, and $4.2 \%$ higher than cob length under high planting density in 2016, 2017, and 2018, respectively (Table 2). Cob number was higher under high planting density than under low planting density under the same mulch and $\mathrm{N}$ fertilizer application rate treatments. Averaged across $\mathrm{N}$ fertilizer application rates, high planting density increased cob number by $66.9,65.7$, and $65.2 \%$ under the no mulch treatment in 2016, 2017, and 2018, respectively, and increased cob number by $65.6,66.2$, and $64.4 \%$ under mulch (Table 2). Compared with high planting density, low planting density increased kernel number per cob by $24.0,23.2$, and $21.3 \%$ under the no mulch treatment in 2016, 2017, and 2018, respectively, and by $22.8,18.8$, and $18.6 \%$ under mulch (Table 2). Because cob number was higher under high planting density, thousand kernel weight was considerably lower under high planting density than under low planting density. Compared with low planting density, high planting density decreased thousand kernel weight by $35.5,34.0$, and $36.1 \%$ under the no mulch treatment in 2016, 2017, and 2018 , respectively, and by $8.8,7.7$, and $9.6 \%$ under mulch (Table 2). Averaged over planting density and $\mathrm{N}$ fertilizer application rate, cob length, kernel number per cob, and thousand kernel weight were higher under mulch than no mulch (Table 2).

\section{Nitrogen uptake at silking and at physiological maturity}

Plant $\mathrm{N}$ uptake at silking and physiological maturity in different organs was affected by mulch, planting density, and $\mathrm{N}$ fertilizer application rate are shown in Figs. 4 and 5. The individual factors of mulch, planting density, and $\mathrm{N}$ fertilizer application rate significantly affected $\mathrm{N}$ uptake by different organs at silking and physiological maturity (Table 3). The interaction between mulch and $\mathrm{N}$ fertilizer rate had a significant effect on $\mathrm{N}$ uptake by different organs at silking and physiological maturity (Table 3). In addition, there was a significant three-way interaction effect among mulch, planting density, and $\mathrm{N}$ fertilizer rate on $\mathrm{N}$ uptake by different organs at silking and physiological maturity, except leaf $\mathrm{N}$ uptake at silking (Table 3). 


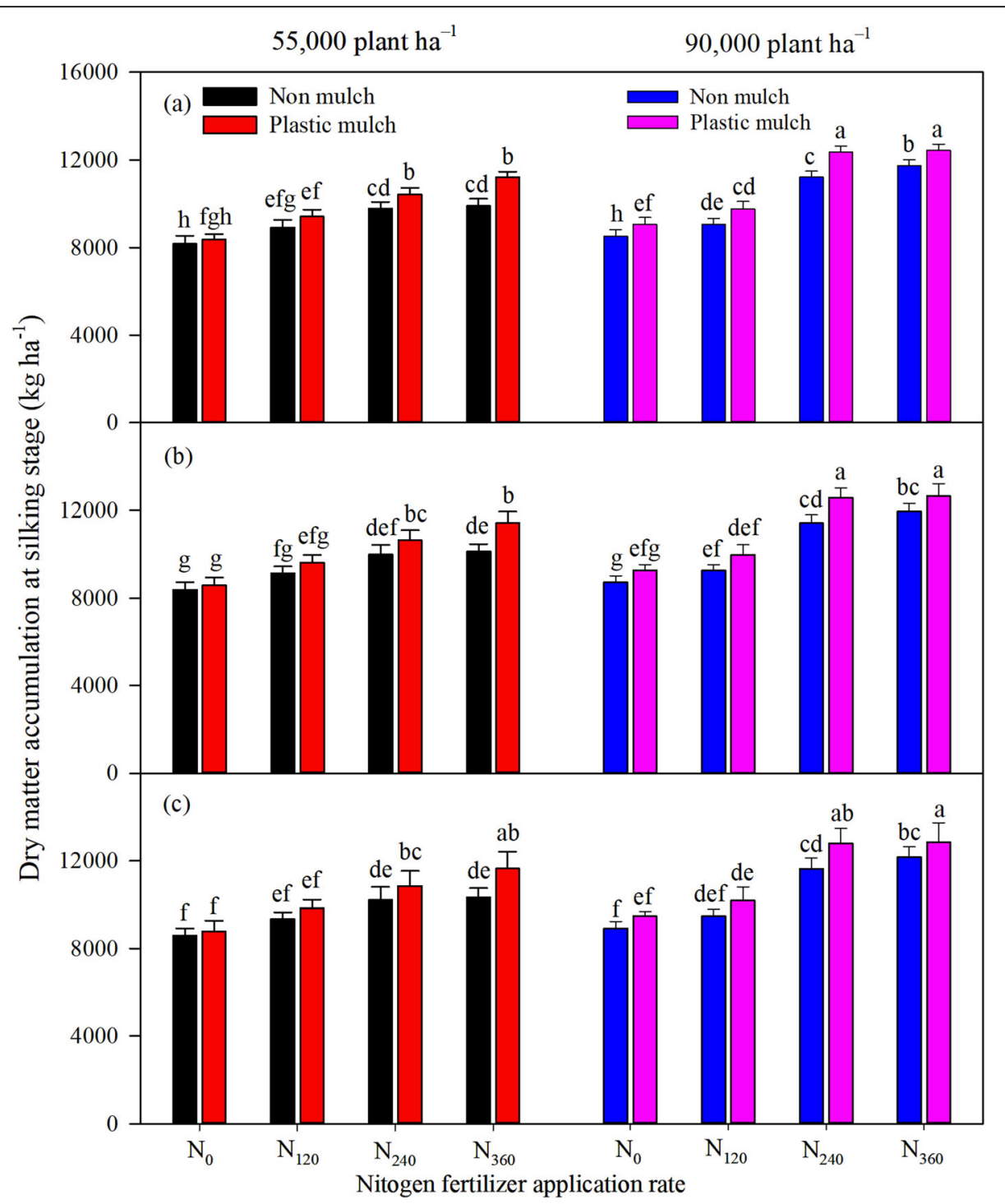

Fig. 1 Effects of mulching (M), planting density (D), and nitrogen fertilizer application rates (N) on maize dry matter accumulation at silking in 2016 (a), 2017 (b), and 2018 (c). The bars represent the mean + one standard error of the mean $(n=4)$. Different letters denote significant differences between treatments (Tukey's HSD post hoc tests, $P<0.05$ ). Note: $\mathrm{N}_{0}, 0 \mathrm{~kg} \mathrm{~N} \mathrm{ha}^{-1} ; \mathrm{N}_{120}, 120 \mathrm{~kg} \mathrm{~N} \mathrm{ha}^{-1} ; \mathrm{N}_{240}, 240 \mathrm{~kg} \mathrm{~N} \mathrm{ha}^{-1} ; \mathrm{N}_{360}, 360 \mathrm{~kg} \mathrm{~N} \mathrm{ha}^{-1}$

Total plant $\mathrm{N}$ uptake increased with increasing $\mathrm{N}$ fertilizer application rate (with or without mulch) at silking, and total plant $\mathrm{N}$ uptake was higher for mulch treatments than for no mulch treatments under the same $\mathrm{N}$ fertilizer application rate (Fig. 4). Averaged over the 3 years of the study, total plant $\mathrm{N}$ uptake at silking ranged from 79 to $149 \mathrm{~kg} \mathrm{~N} \mathrm{ha}^{-1}$ with no mulch and from 76 to $178 \mathrm{~kg} \mathrm{~N} \mathrm{ha}^{-1}$ with mulch (Fig. 4). Similarly, total plant $\mathrm{N}$ uptake values with mulch (ranging from 82 to $180 \mathrm{~kg}$ $\mathrm{Nha}^{-1}$ ) were higher than observed with no mulch (ranging from 94 to $194 \mathrm{~kg} \mathrm{~N} \mathrm{ha}^{-1}$ ) at physiological maturity (Fig. 5). The $\mathrm{N}$ uptake by different organs at silking ranked as leaf $>$ grain $>$ stem $>$ cob, but at physiological maturity the ranking changed to grain $>$ leaf $>$ stem $>$ cob at physiological maturity stage (Figs. 4, 5). In general, the highest total plant $\mathrm{N}$ uptake was observed for the $\mathrm{N}_{360}$ treatment under all mulching and planting density treatments (Figs. 4, 5). Compared with the no $\mathrm{N}$ fertilizer $\left(\mathrm{N}_{0}\right)$ treatment, $\mathrm{N}_{120}, \mathrm{~N}_{240}$, and $\mathrm{N}_{360}$ increased total plant $\mathrm{N}$ uptake by 50.0, 85.8 and $95.0 \%$ at silking, respectively, and by $54.3,90.0$ and $95.6 \%$ at physiological maturity when averaged across mulch and planting density treatments and years (Figs. 4, 5). In contrast, total plant $\mathrm{N}$ uptake under high planting density was higher than under low planting density. Total plant $\mathrm{N}$ uptake was more sensitive to mulch than to planting density. Averaged across planting density, $\mathrm{N}$ fertilizer application rate, and years, mulch increased total plant $\mathrm{N}$ uptake by $13 \%$ at silking and by $8.2 \%$ at physiological maturity (Figs. 4, 5). Averaged across mulch, $\mathrm{N}$ fertilizer 


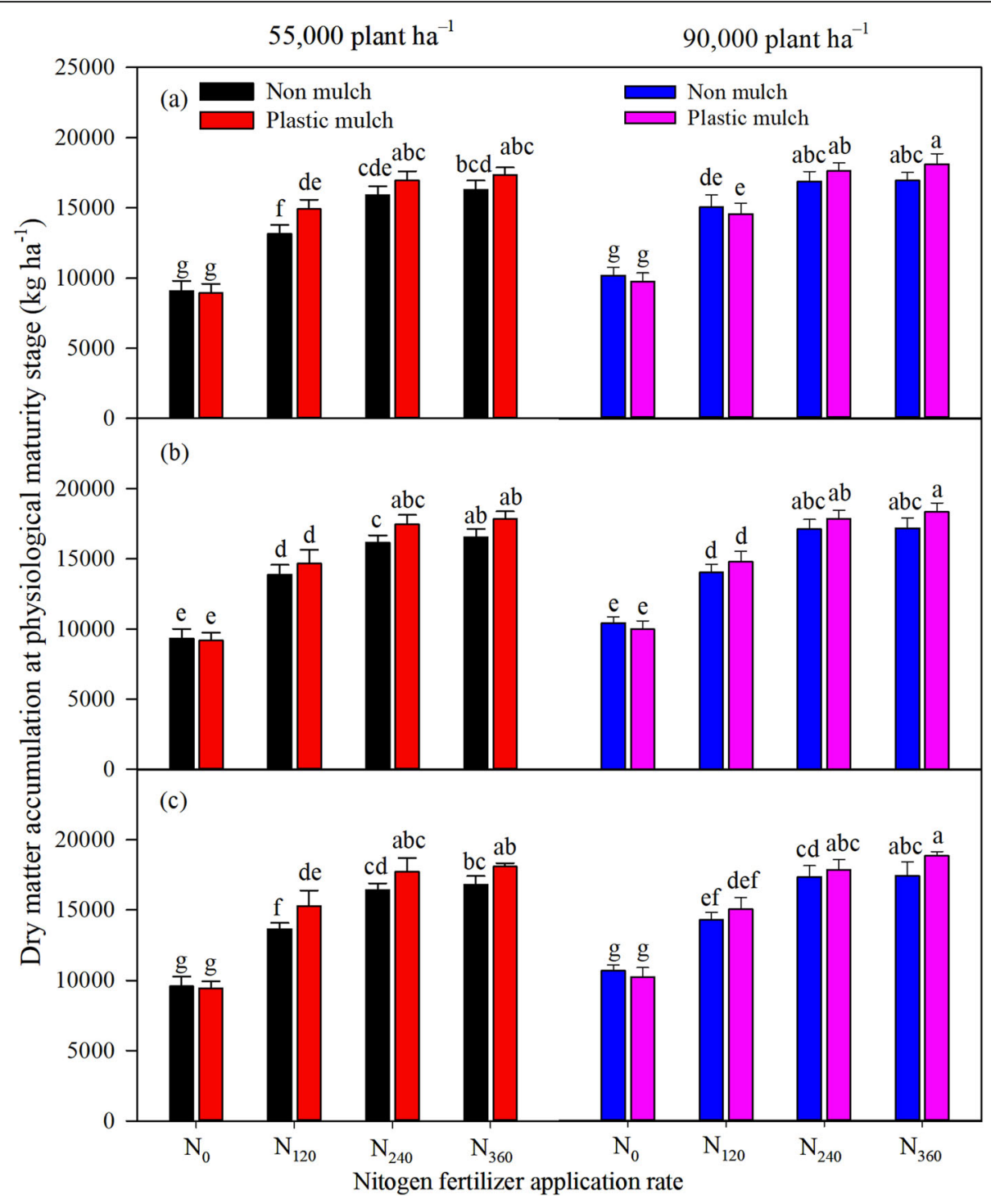

Fig. 2 Effects of mulching (M), planting density (D), and nitrogen fertilizer application rates $(N)$ on maize dry matter accumulation at physiological maturity in 2016 (a), 2017 (b), and 2018 (c). The bars represent the mean + one standard error of the mean $(n=4)$. Different letters denote

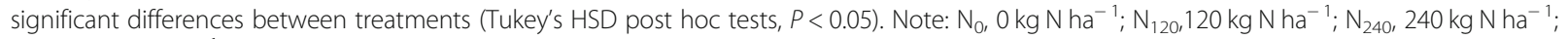
$\mathrm{N}_{360}, 360 \mathrm{~kg} \mathrm{~N} \mathrm{ha}^{-1}$

application rate, and years, high plant population density increased total plant $\mathrm{N}$ uptake by $3.5 \%$ at silking and by $3.4 \%$ at physiological maturity (Figs. 4,5 ).

\section{Translocated $\mathrm{N}$ from vegetative organs and $\mathrm{N}$ translocation efficiency}

A significant three-way interaction among mulch, planting density, and $\mathrm{N}$ fertilizer application rate, and significant two-way interactions between mulch and $\mathrm{N}$ fertilizer application rate and between planting density and $\mathrm{N}$ fertilizer application rate were observed for $\mathrm{N}$ translocation and $\mathrm{N}$ translocation efficiency in different maize organs. The mulch and planting density treatments had no significant interaction for $\mathrm{N}$ translocation and $\mathrm{N}$ translocation efficiency in the cob (Table 4). The individual factors of mulch, planting density, and $\mathrm{N}$ fertilizer application rate significantly affected $\mathrm{N}$ translocation and $\mathrm{N}$ translocation efficiency in different plant organs (Table 4). The total $\mathrm{N}$ translocation from vegetative organs was reduced with the increase of planting density. Total $\mathrm{N}$ translocation under high planting density averaged over $\mathrm{N}$ fertilizer application rates and years was 10.9 and $4.8 \%$ lower than observed under low planting density with and without mulch, respectively (Fig. 6). The $\mathrm{N}$ translocation from different organs ranked as leaf $>$ stem $>$ cob in all 


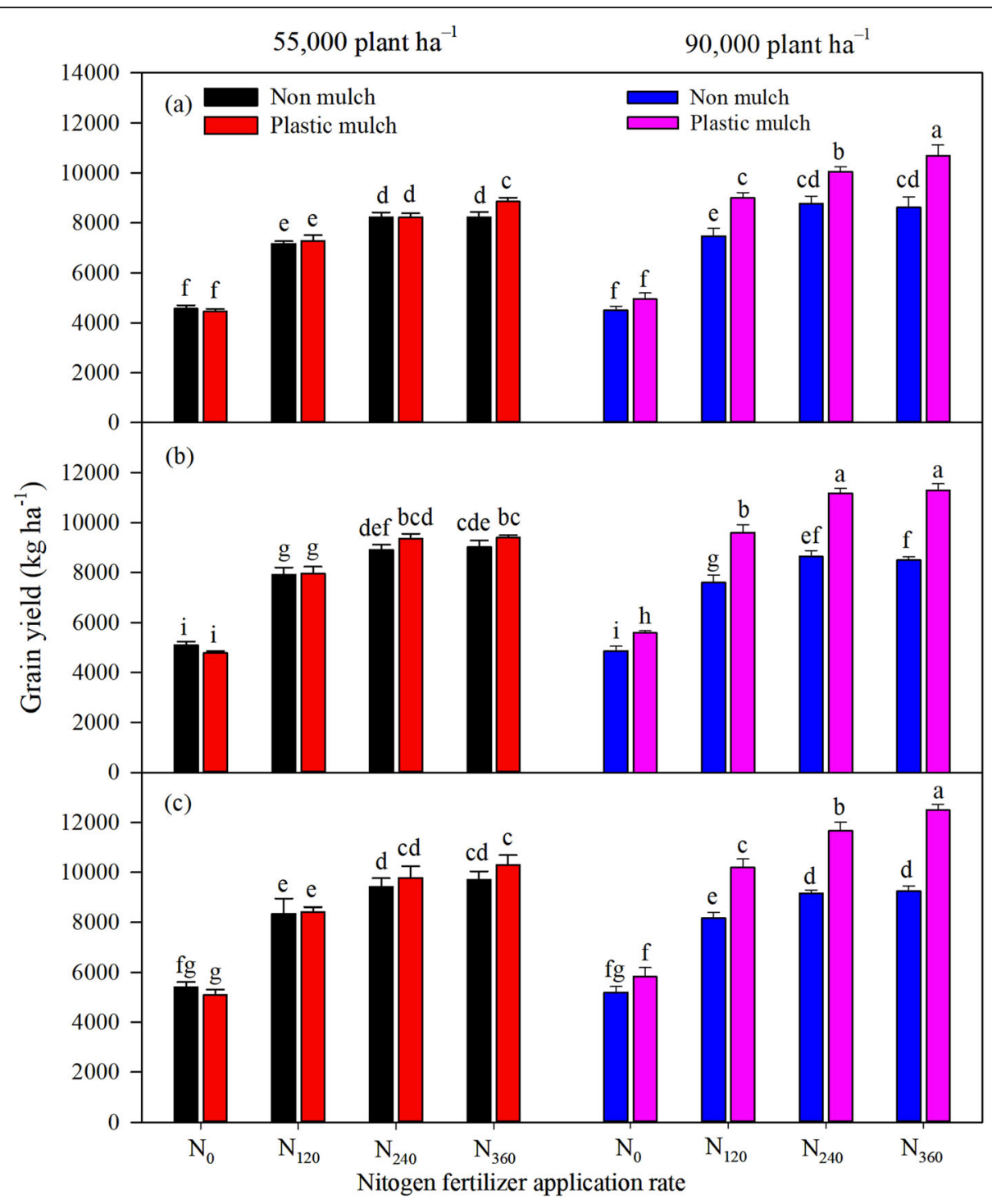

Fig. 3 Effects of mulching, plant density, and nitrogen fertilizer application rates on maize grain yield in 2016 (a), 2017 (b), and 2018 (c). The bars represent the mean + one standard error of the mean $(n=4)$. Different letters denote significant differences between treatments (Tukey's HSD post hoc tests, $P<0.05)$. Note: $\mathrm{N}_{0}, 0 \mathrm{~kg} \mathrm{~N} \mathrm{ha}^{-1} ; \mathrm{N}_{120}, 120 \mathrm{~kg} \mathrm{~N} \mathrm{ha}^{-1} ; \mathrm{N}_{240}, 240 \mathrm{~kg} \mathrm{~N} \mathrm{ha}^{-1} ; \mathrm{N}_{360}, 360 \mathrm{~kg} \mathrm{~N} \mathrm{ha}^{-1}$

treatments (Fig. 6). Averaged across all factors, the highest $\mathrm{N}$ translocation was observed for leaf, which was $59.4 \%\left(14.6 \mathrm{~kg} \mathrm{~N} \mathrm{ha}^{-1}\right)$ and $88.7 \%\left(21.9 \mathrm{~kg} \mathrm{~N} \mathrm{ha}^{-1}\right)$ higher than that for stem and cob, respectively (Fig. 6). Leaf $\mathrm{N}$ translocation increased with increasing $\mathrm{N}$ fertilizer application rate, but $\mathrm{N}$ translocation in stem and cob did not follow this pattern. Averaged over mulch and planting density treatments and years, leaf $\mathrm{N}$ translocation for $\mathrm{N}_{360}$ was $8.7 \%\left(3 \mathrm{~kg} \mathrm{Nha}^{-1}\right), 35.1 \%\left(12 \mathrm{~kg} \mathrm{Nha}^{-1}\right)$, and $68.3 \%\left(23.5 \mathrm{~kg} \mathrm{Nha}^{-1}\right)$ higher than that observed for $\mathrm{N}_{240}, \mathrm{~N}_{120}$, and $\mathrm{N}_{0}$, respectively (Fig. 6).

Averaged over planting density and year, leaf $\mathrm{N}$ translocation efficiency increased with increasing $\mathrm{N}$ fertilizer application rate with the no mulch treatment, but leaf $\mathrm{N}$ translocation efficiency initially increased and then decreased with increasing $\mathrm{N}$ fertilizer application rate under the mulch treatment (Fig. 7). Considering all treatments, leaf $\mathrm{N}$ translocation efficiency ranged from 9.5 to $24.5 \%$, stem $\mathrm{N}$ translocation efficiency ranged from 2.6 to $13.5 \%$, and cob $\mathrm{N}$ translocation efficiency was ranged from 0.4 to $3.3 \%$ (Fig. 7). Averaged over N fertilizer application rate and year, plastic mulch increased leaf $\mathrm{N}$ translocation efficiency by $16.3 \%$ under low planting density and by $18.8 \%$ under high planting density (Fig. 7). Averaged over $\mathrm{N}$ fertilizer application rate and year, mulch increased leaf $\mathrm{N}$ translocation efficiency by $15 \%$ over the no mulch treatment, but decreased stem $\mathrm{N}$ translocation efficiency by $43 \%$ and decreased cob $\mathrm{N}$ translocation efficiency by $15.2 \%$ (Fig. 7). The mean vegetative organ $\mathrm{N}$ translocation efficiency 
Table 2 Maize cob length, cob number, kernel number per cob, and thousand kernel weight as affected by mulch, nitrogen fertilizer application rate, and planting density ( $D_{\llcorner}: 55,000$ seeds ha ${ }^{-1} ; D_{H}: 90,000$ seeds ha $\left.{ }^{-1}\right)$ in 2015, 2016, and 2017

\begin{tabular}{|c|c|c|c|c|c|c|c|c|c|c|}
\hline \multirow[t]{2}{*}{ Year } & \multirow[t]{2}{*}{ Mulch } & \multirow[t]{2}{*}{ Nitrogen } & \multicolumn{2}{|c|}{ Cob length $(\mathrm{cm})$} & \multicolumn{2}{|c|}{$\begin{array}{l}\text { Cob number } \\
\left(\mathrm{ha}^{-1}\right)\end{array}$} & \multicolumn{2}{|c|}{ Kernel number per cob } & \multicolumn{2}{|c|}{ Thousand kernel weight ( $\mathrm{g}$ ) } \\
\hline & & & $D_{L}$ & $\mathrm{D}_{\mathrm{H}}$ & $D_{\mathrm{L}}$ & $\mathrm{D}_{\mathrm{H}}$ & $D_{L}$ & $D_{H}$ & $D_{L}$ & $\mathrm{D}_{\mathrm{H}}$ \\
\hline \multirow[t]{8}{*}{2015} & \multirow[t]{4}{*}{ No } & $\mathrm{N}_{0}$ & 14.6 & 15.3 & 42,997 & 77,757 & 470 & 385 & 223 & 149 \\
\hline & & $\mathrm{N}_{120}$ & 16.6 & 16.3 & 48,703 & 79,281 & 555 & 437 & 276 & 211 \\
\hline & & $\mathrm{N}_{240}$ & 17.3 & 16.5 & 48,894 & 79,500 & 571 & 469 & 315 & 222 \\
\hline & & $\mathrm{N}_{360}$ & 17.2 & 16.5 & 49,023 & 79,953 & 581 & 465 & 299 & 240 \\
\hline & \multirow[t]{4}{*}{ Yes } & $\mathrm{N}_{0}$ & 15.3 & 14.3 & 45,143 & 76,345 & 473 & 418 & 214 & 155 \\
\hline & & $\mathrm{N}_{120}$ & 17.2 & 16.8 & 48,642 & 79,087 & 583 & 461 & 253 & 248 \\
\hline & & $\mathrm{N}_{240}$ & 17.6 & 16.9 & 49,284 & 81,562 & 617 & 485 & 271 & 255 \\
\hline & & $N_{360}$ & 17.5 & 16.8 & 49,112 & 81,349 & 613 & 497 & 276 & 265 \\
\hline \multirow[t]{8}{*}{2016} & \multirow[t]{4}{*}{ No } & $\mathrm{N}_{0}$ & 15.4 & 15.6 & 45,114 & 77,496 & 484 & 395 & 234 & 157 \\
\hline & & $\mathrm{N}_{120}$ & 17.0 & 16.5 & 48,892 & 80,244 & 563 & 447 & 290 & 221 \\
\hline & & $\mathrm{N}_{240}$ & 17.6 & 16.8 & 49,151 & 79,772 & 579 & 482 & 330 & 233 \\
\hline & & $N_{360}$ & 17.4 & 16.6 & 48,679 & 80,270 & 583 & 469 & 301 & 252 \\
\hline & \multirow[t]{4}{*}{ Yes } & $\mathrm{N}_{0}$ & 15.9 & 15.4 & 45,304 & 77,291 & 478 & 444 & 225 & 163 \\
\hline & & $\mathrm{N}_{120}$ & 18.0 & 17.1 & 49,151 & 80,691 & 582 & 459 & 266 & 261 \\
\hline & & $\mathrm{N}_{240}$ & 18.1 & 17.2 & 49,705 & 82,303 & 613 & 509 & 285 & 268 \\
\hline & & $N_{360}$ & 17.8 & 17.2 & 50,029 & 82,422 & 594 & 495 & 289 & 278 \\
\hline \multirow[t]{8}{*}{2017} & \multirow[t]{4}{*}{ No } & $N_{0}$ & 16.0 & 15.7 & 44,857 & 78,187 & 492 & 398 & 246 & 165 \\
\hline & & $\mathrm{N}_{120}$ & 16.9 & 16.7 & 49,651 & 81,211 & 553 & 448 & 305 & 232 \\
\hline & & $\mathrm{N}_{240}$ & 17.6 & 16.6 & 49,105 & 79,865 & 576 & 484 & 347 & 245 \\
\hline & & $N_{360}$ & 17.4 & 16.5 & 49,166 & 79,301 & 591 & 494 & 335 & 264 \\
\hline & \multirow[t]{4}{*}{ Yes } & $\mathrm{N}_{0}$ & 15.4 & 15.2 & 45,633 & 76,469 & 484 & 447 & 236 & 171 \\
\hline & & $\mathrm{N}_{120}$ & 18.0 & 16.9 & 49,764 & 80,041 & 590 & 468 & 279 & 274 \\
\hline & & $\mathrm{N}_{240}$ & 17.7 & 17.0 & 49,960 & 81,762 & 615 & 501 & 299 & 281 \\
\hline & & $N_{360}$ & 18.1 & 16.9 & 49,391 & 81,838 & 622 & 525 & 302 & 292 \\
\hline \multicolumn{11}{|c|}{ Treatment effect ( $P$ values) ${ }^{a}$} \\
\hline \multicolumn{3}{|c|}{ Year $(Y)$} & \multicolumn{2}{|l|}{$<0.001$} & \multicolumn{2}{|l|}{ ns } & \multicolumn{2}{|l|}{0.008} & \multicolumn{2}{|l|}{$<0.001$} \\
\hline \multicolumn{3}{|c|}{ Mulch (M) } & $<0.001$ & & ns & & $<0.001$ & & $<0.001$ & \\
\hline Nitrog & (N) & & $<0.001$ & & $<0.001$ & & $<0.001$ & & $<0.001$ & \\
\hline Densit & & & $<0.001$ & & $<0.001$ & & $<0.001$ & & $<0.001$ & \\
\hline$Y * M$ & & & ns & & ns & & ns & & ns & \\
\hline$Y * N$ & & & ns & & ns & & ns & & ns & \\
\hline$Y * D$ & & & ns & & ns & & ns & & ns & \\
\hline$M * N$ & & & 0.01 & & ns & & ns & & $<0.001$ & \\
\hline$M * D$ & & & ns & & ns & & ns & & ns & \\
\hline$N * D$ & & & ns & & ns & & $<0.001$ & & $<0.001$ & \\
\hline$Y^{*} M$ & & & ns & & ns & & ns & & ns & \\
\hline$Y * M$ & & & ns & & ns & & ns & & ns & \\
\hline$Y^{*} N$ & & & ns & & ns & & ns & & ns & \\
\hline$M * N$ & & & ns & & ns & & 0.006 & & ns & \\
\hline$Y^{*} M$ & $\checkmark * D$ & & ns & & ns & & ns & & ns & \\
\hline
\end{tabular}

${ }^{a}$ ns Not significant $(P>0.05)$ 

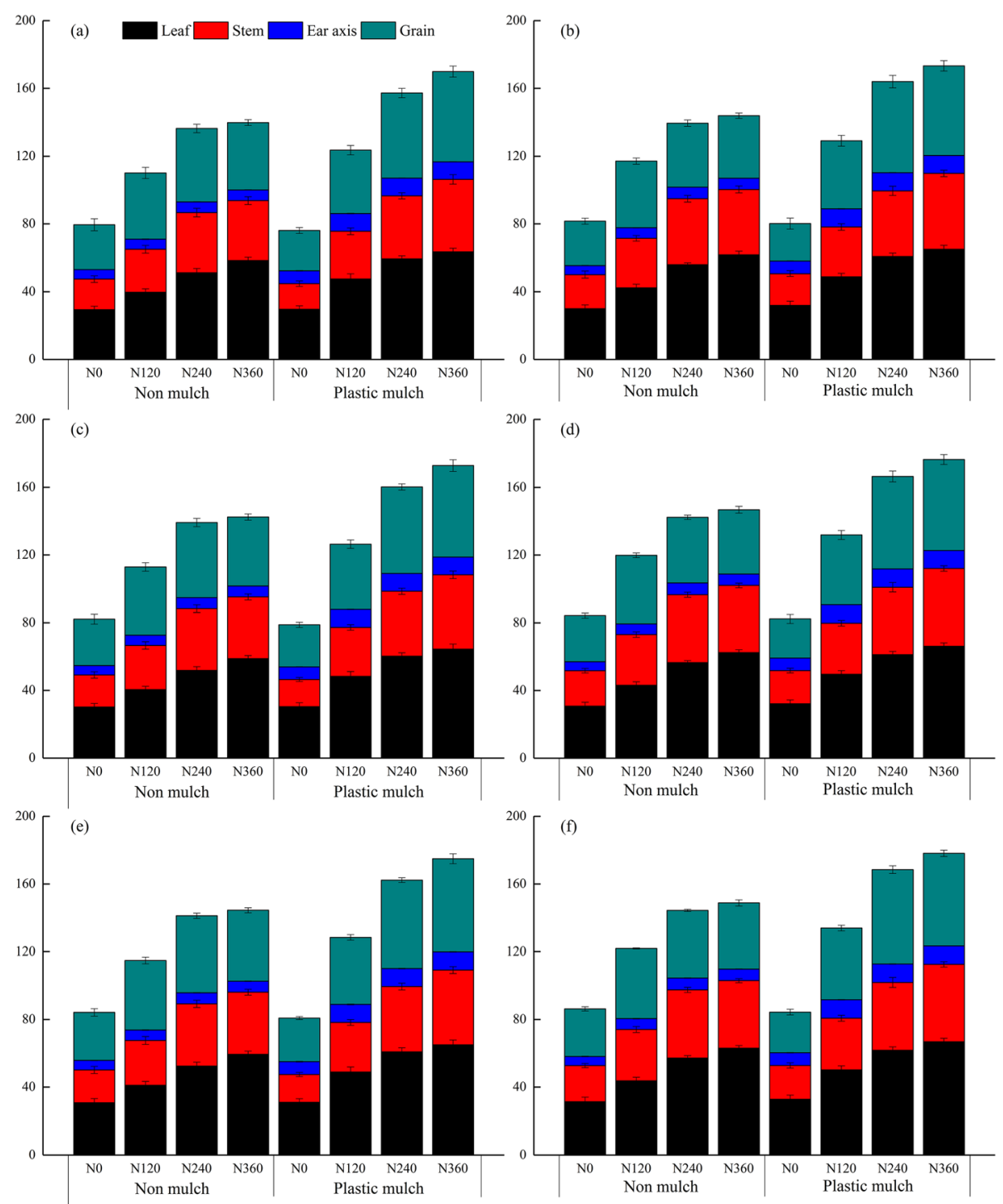

Fig. 4 Plant nitrogen uptake at silking by different maize organs as affected by mulching (no mulch, plastic mulch), planting density (55,000 seeds $\mathrm{ha}^{-1}, 90,000$ seeds ha $\left.{ }^{-1}\right)$, and $\mathrm{N}$ fertilizer application rate $\left(\mathrm{N}_{0}, 0 \mathrm{~kg} \mathrm{~N} \mathrm{ha}^{-1} ; \mathrm{N}_{120}, 120 \mathrm{~kg} \mathrm{~N} \mathrm{ha}^{-1} ; \mathrm{N}_{240}, 240 \mathrm{~kg} \mathrm{~N} \mathrm{ha}^{-1} ; \mathrm{N}_{360}, 360 \mathrm{~kg} \mathrm{~N} \mathrm{ha}^{-1}\right)$ in $2016(\mathbf{a}, \mathbf{b})$, $2017(\mathbf{c}, \mathbf{d})$, and 2018 (e, f). Bars represent the mean + one standard error of the mean $(n=4)$

averaged over mulch, planting density, and $\mathrm{N}$ fertilizer application rate treatments decreased in the order of leaf $>$ stem > cob (Fig. 7).

\section{Nitrogen harvest index and nitrogen use efficiency}

The individual factors of mulch and $\mathrm{N}$ fertilizer application rate significantly affected $\mathrm{N}$ assimilation amount (NAAS), and there was a significant interaction between mulch and $\mathrm{N}$ fertilizer application rate on NAAS (Table 5). The NAAS ranged from 29.9 to $74.1 \mathrm{~kg} \mathrm{ha}^{-1}$ for the low planting density and from 31.9 to $76.3 \mathrm{~kg}$ $\mathrm{ha}^{-1}$ in the high planting density among the different treatment groups (Table 5). Averaged over mulch, planting density, and year, the highest value of NAAS was obtained with $\mathrm{N}_{240}\left(73.2 \mathrm{~kg} \mathrm{ha}^{-1}\right)$, which was $49,15.6$, and $4.5 \%$ higher than NASS obtained with $\mathrm{N}_{0}, \mathrm{~N}_{120}$, and $\mathrm{N}_{360}$ (Table 5).

The individual factors of mulch, planting density, and $\mathrm{N}$ fertilizer application rate had significant effects on $\mathrm{N}$ harvest index (NHI), $\mathrm{N}$ use efficiency (NUE), $\mathrm{N}$ apparent recovery efficiency (NRE), and partial factor productivity of the fertilizer (PFP) (Table 5). There was a significant three-way interaction among mulch, planting density, and $\mathrm{N}$ fertilizer application rate and significant two-way interactions between each pair of factors for NHI, NUE, and PFP (Table 5). The NHI for the mulch treatment was lower than observed for the no mulch treatment under the same $\mathrm{N}$ fertilizer application rate (Table 5). 


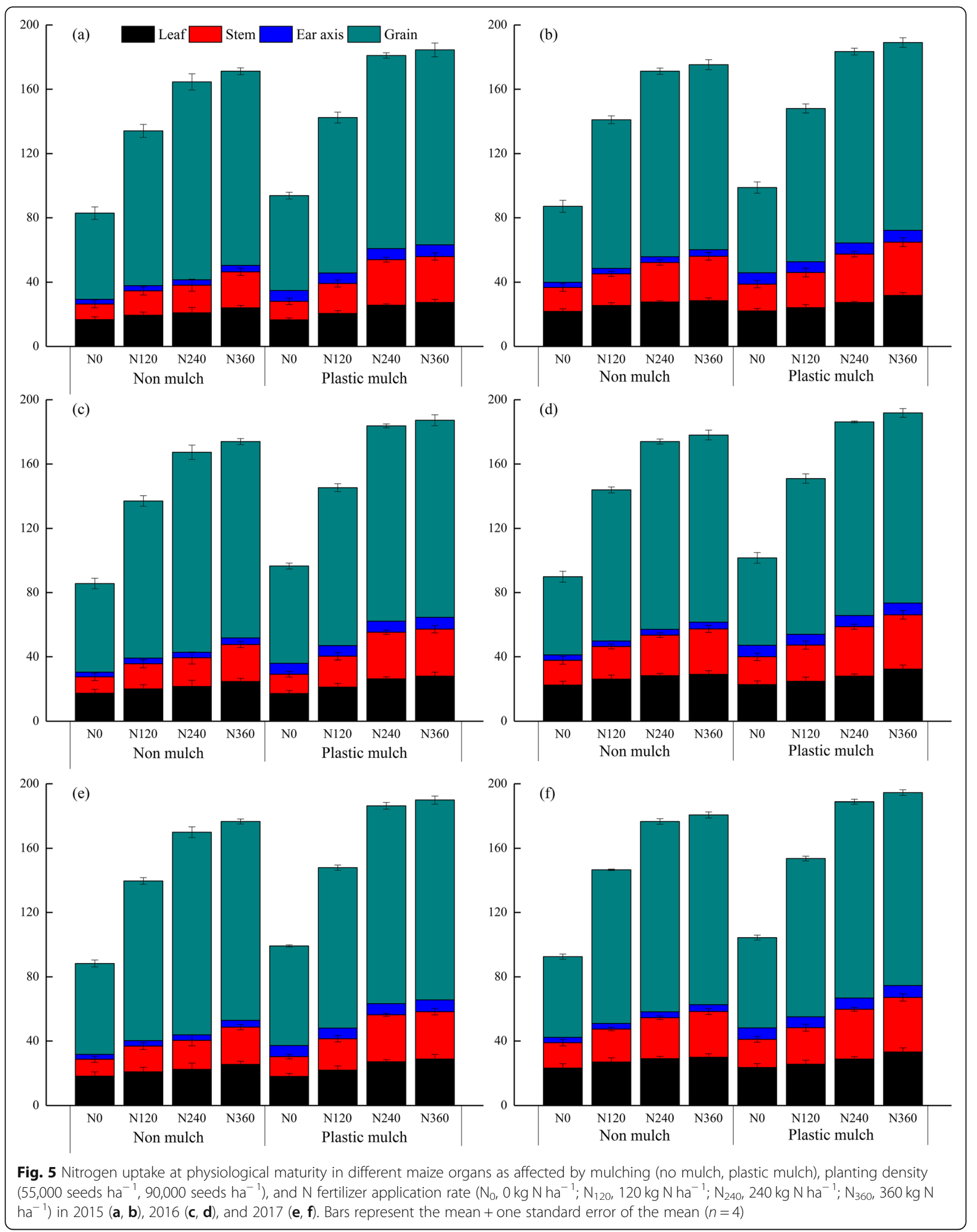


Table 3 Treatment effects ( $p$ values) for the nitrogen uptake by different maize organs at silking and physiological maturity for four fixed factors (year, mulch, nitrogen fertilizer application rate, and planting density)

\begin{tabular}{|c|c|c|c|c|c|c|c|c|}
\hline \multirow[t]{2}{*}{ Sources } & \multicolumn{4}{|c|}{ Nitrogen uptake at the silking } & \multicolumn{4}{|c|}{ Nitrogen uptake at physiological maturity } \\
\hline & Leaf & Stem & Cob & Grain & Leaf & Stem & Cob & Grain \\
\hline Year $(Y)$ & 0.003 & 0.001 & $<0.001$ & $<0.001$ & 0.002 & ns & $<0.001$ & $<0.001$ \\
\hline Mulch (M) & $<0.001$ & $<0.001$ & $<0.001$ & $<0.001$ & $<0.001$ & $<0.001$ & $<0.001$ & $<0.001$ \\
\hline Nitrogen (N) & $<0.001$ & $<0.001$ & $<0.001$ & $<0.001$ & $<0.001$ & $<0.001$ & $<0.001$ & $<0.001$ \\
\hline Density (D) & $<0.001$ & $<0.001$ & $<0.001$ & $<0.001$ & $<0.001$ & $<0.001$ & $<0.001$ & $<0.001$ \\
\hline$Y * M$ & ns & ns & ns & ns & ns & ns & ns & ns \\
\hline$Y * N$ & ns & ns & ns & ns & ns & ns & ns & ns \\
\hline$Y * D$ & ns & ns & ns & ns & ns & ns & ns & ns \\
\hline$M * N$ & $<0.001$ & $<0.001$ & $<0.001$ & $<0.001$ & $<0.001$ & $<0.001$ & $<0.001$ & $<0.001$ \\
\hline$M * D$ & 0.034 & ns & ns & $<0.001$ & 0.006 & 0.002 & ns & 0.001 \\
\hline$N * D$ & ns & ns & $<0.001$ & 0.007 & ns & ns & $<0.001$ & 0.012 \\
\hline$Y * M * N$ & ns & ns & ns & ns & ns & ns & ns & ns \\
\hline$Y * M * D$ & ns & ns & ns & ns & ns & ns & ns & ns \\
\hline$Y * N * D$ & ns & ns & ns & ns & ns & ns & ns & ns \\
\hline$M * N * D$ & ns & 0.05 & 0.005 & $<0.001$ & 0.01 & 0.008 & 0.007 & 0.028 \\
\hline$Y * M * N * D$ & ns & ns & ns & ns & ns & ns & ns & ns \\
\hline
\end{tabular}

Note: $n s$ Not significant $(p>0.05)$

Averaged over $\mathrm{N}$ fertilizer application rate and year, mulch decreased NHI by $7.2 \%$ compared with the no mulch treatment for the low planting density and by $3.3 \%$ in high planting density (Table 5). However, NHI did not increase with increasing planting density. Averaged over mulch, $\mathrm{N}$ fertilizer application rate, and year,
NHI for the high planting density was $9.3 \%$ lower than observed for the low planting density (Table 5). Averaged over $\mathrm{N}$ fertilizer application rate and year, mulch increased NUE by $6.5 \%$ for the low planting density and by $22 \%$ for the high planting density (Table 5 ). The NUE decreased with increasing $\mathrm{N}$ fertilizer application rate,

Table 4 Treatment effects ( $p$ values) for nitrogen translocation and nitrogen translocation efficiency of different maize organs, for four fixed factors (year, mulch, nitrogen fertilizer application rate, and planting density)

\begin{tabular}{|c|c|c|c|c|c|c|}
\hline \multirow[t]{2}{*}{ Sources } & \multicolumn{3}{|c|}{ Nitrogen translocation } & \multicolumn{3}{|c|}{ Nitrogen translocation efficiency } \\
\hline & Leaf & Stem & Cob & Leaf & Stem & Cob \\
\hline Year $(Y)$ & ns & ns & 0.018 & 0.015 & ns & ns \\
\hline Mulch (M) & $<0.001$ & $<0.001$ & $<0.001$ & $<0.001$ & $<0.001$ & $<0.001$ \\
\hline Nitrogen $(\mathrm{N})$ & $<0.001$ & $<0.001$ & $<0.001$ & $<0.001$ & $<0.001$ & $<0.001$ \\
\hline Density (D) & $<0.001$ & $<0.001$ & 0.009 & $<0.001$ & $<0.001$ & $<0.001$ \\
\hline$Y * M$ & ns & ns & ns & ns & ns & ns \\
\hline$Y * N$ & ns & ns & ns & ns & ns & ns \\
\hline$Y * D$ & ns & ns & ns & ns & ns & ns \\
\hline$M * N$ & $<0.001$ & $<0.001$ & $<0.001$ & $<0.001$ & $<0.001$ & $<0.001$ \\
\hline$M * D$ & ns & 0.018 & ns & $<0.001$ & $<0.001$ & ns \\
\hline$N * D$ & $<0.001$ & 0.012 & $<0.001$ & $<0.001$ & $<0.001$ & $<0.001$ \\
\hline$Y * M * N$ & ns & ns & ns & ns & ns & ns \\
\hline$Y * M * D$ & ns & ns & ns & ns & ns & ns \\
\hline$Y * N * D$ & ns & ns & ns & ns & ns & ns \\
\hline$M * N * D$ & 0.001 & $<0.001$ & 0.002 & 0.009 & 0.002 & $<0.001$ \\
\hline$Y * M * N * D$ & ns & ns & ns & ns & ns & ns \\
\hline
\end{tabular}

Note: $n s$ Not significant $(p>0.05)$ 

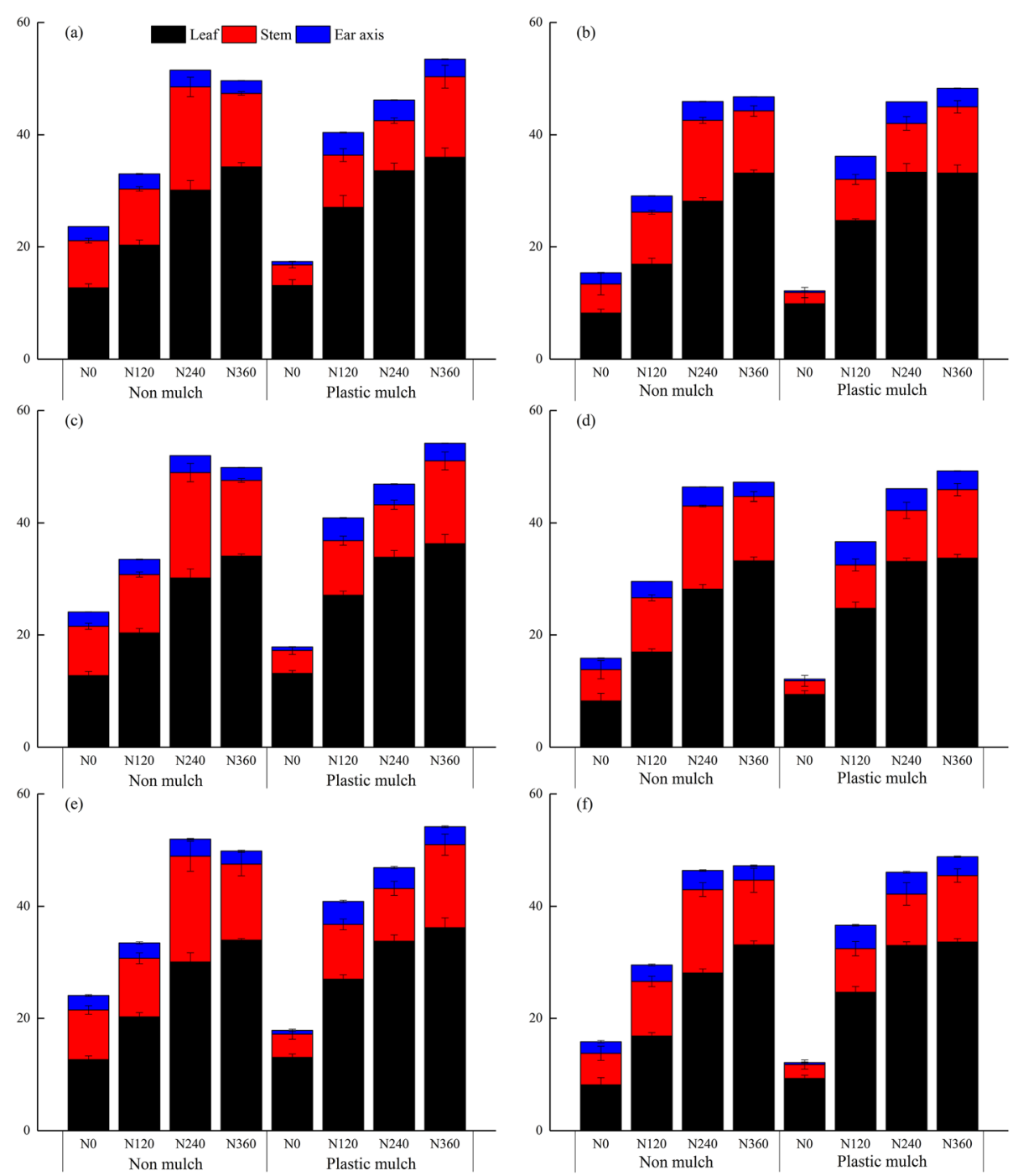

Fig. 6 Nitrogen translocation in different maize organs (leaf, stem and cob) from silking to physiological maturity as affected by mulching (no mulch, plastic mulch), planting density (55,000 seeds ha ${ }^{-1}, 90,000$ seeds ha $\left.{ }^{-1}\right)$, and $\mathrm{N}$ fertilizer application rate $\left(\mathrm{N}_{0}, 0 \mathrm{~kg} \mathrm{~N} \mathrm{ha}{ }^{-1} ; \mathrm{N}_{120}, 120 \mathrm{~kg} \mathrm{~N}\right.$ $h^{-1} ; N_{240}, 240 \mathrm{~kg} \mathrm{~N} \mathrm{ha}^{-1} ; \mathrm{N}_{360}, 360 \mathrm{~kg} \mathrm{~N} \mathrm{ha}^{-1}$ ) in 2015 (a, b), 2016 (c, d), and 2017 (e, f). Bars represent the mean + one standard error of the mean $(n=4)$

and the $\mathrm{N}_{120}$ treatment had the highest NUE among all $\mathrm{N}$ fertilizer application rates (Table 5). Averaged over mulch treatment and year, the $\mathrm{N}_{120}$ treatment increased NUE by 42.3 and $62.0 \%$ over the NUE observed for the $\mathrm{N}_{240}$ and $\mathrm{N}_{360}$ treatments, respectively, at the low planting density, and by 43.4 and $60.0 \%$ at the high planting density (Table 5). Averaged over mulch, $\mathrm{N}$ fertilizer application rate, and year, NRE was similar for the high planting density $\left(34.8 \mathrm{~kg} \mathrm{~kg}^{-1}\right)$ and low planting density $\left(34.2 \mathrm{~kg} \mathrm{~kg}^{-1}\right)$, but PFP was higher for the high planting density $\left(25.4 \mathrm{~kg} \mathrm{~kg}^{-1}\right)$ than for the low planting density $\left(22.5 \mathrm{~kg} \mathrm{~kg}^{-1}\right)$ (Table 5). Averaged over $\mathrm{N}$ fertilizer application rate and planting density, mulch increased PFP by $13.9 \%$ in $2016,14.5 \%$ in 2017 , and $13.4 \%$ in 2018 compared with no mulch, (Table 5).

\section{Discussion}

Mulch with high planting density and increased nitrogen fertilizer application rate can increase maize grain yield and dry matter accumulation

In this study, we analyzed the influence of plastic mulch, planting density, and $\mathrm{N}$ fertilizer application rate and their interactions on maize aboveground dry matter accumulation and yield. This is different from previous studies that only considered the interaction of two factors on maize dry matter accumulation and yield [39-41]. In the present study, there was a significant three-way interaction among mulch, planting density, and $\mathrm{N}$ fertilizer and two-way interactions among each of the pairs of the three factors on maize grain yield (Table 1). The increases in maize grain yield due to mulch treatment 


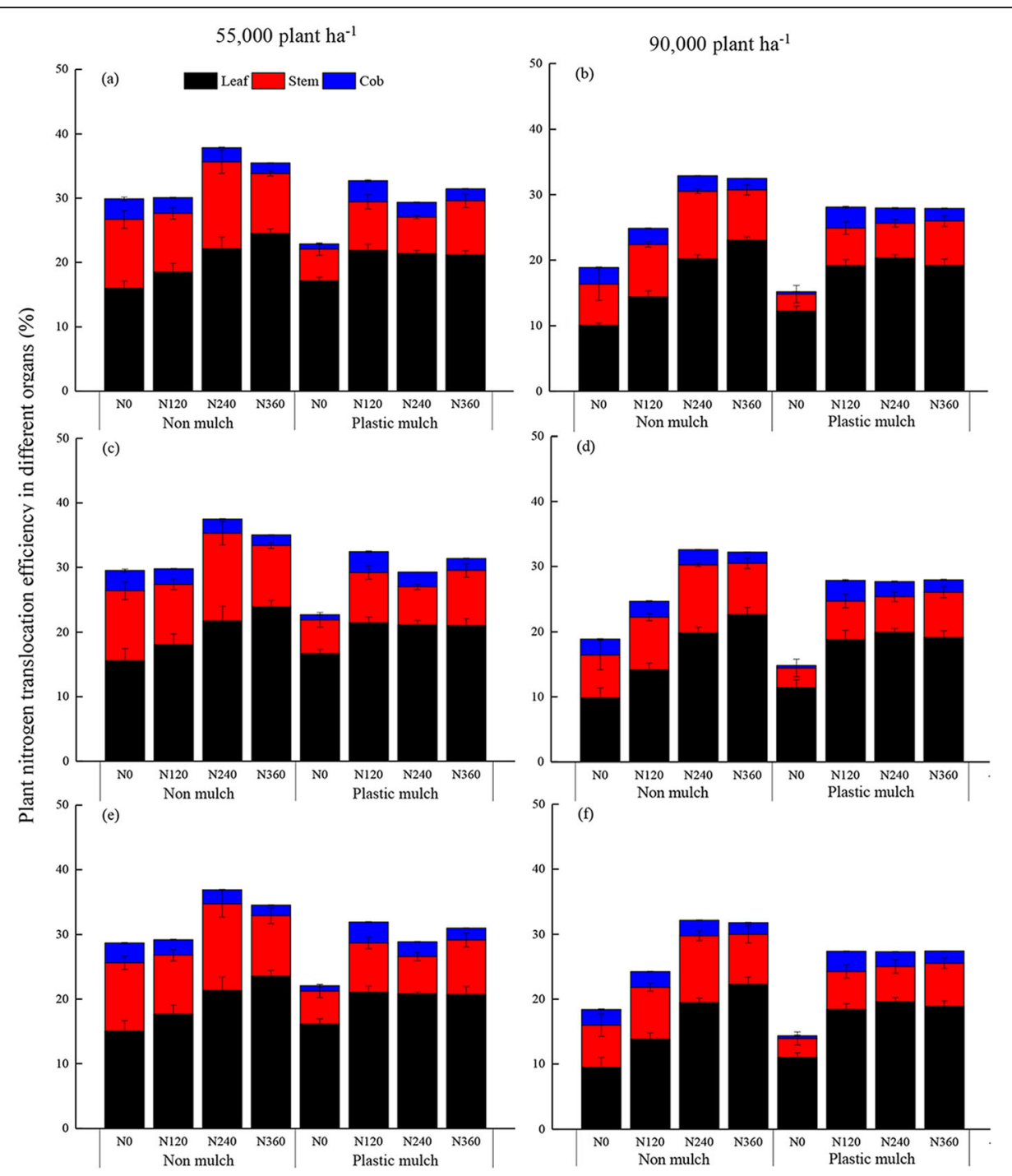

Fig. 7 Nitrogen translocation efficiency in different maize organs (leaf, stem and cob) from silking to physiological maturity as affected by mulching (no mulch, plastic mulch), planting density $\left(55,000\right.$ seeds ha ${ }^{-1}, 90,000$ seeds ha $\left.{ }^{-1}\right)$, and $\mathrm{N}$ fertilizer application rate $\left(\mathrm{N}_{0}, 0 \mathrm{~kg} \mathrm{~N} \mathrm{ha}^{-1} ; \mathrm{N}_{120}, 120 \mathrm{~kg} \mathrm{~N} \mathrm{ha}^{-1}\right.$; $\left.\mathrm{N}_{240}, 240 \mathrm{~kg} \mathrm{~N} \mathrm{ha}^{-1} ; \mathrm{N}_{360}, 360 \mathrm{~kg} \mathrm{~N} \mathrm{ha}^{-1}\right)$ in $2015(\mathbf{a}, \mathbf{b}), 2016(\mathbf{c}, \mathbf{d})$, and $2017(\mathbf{e}, \mathbf{f})$. Bars represent the mean + one standard error of the mean $(n=4)$

were greater under high planting density than under low planting density. Similarly, increasing planting density can increase maize grain yield, but the effect is greater when plastic mulch is used than when no mulch is present. Maize grain yield increased with increasing $\mathrm{N}$ fertilizer application rate, but the rate of increase decreased with increasing $\mathrm{N}$ fertilizer application rate (Fig. S1). This result suggests that plastic film mulch combined with moderate $\mathrm{N}$ fertilizer application rate under high planting density conditions will be an optimal strategy to promote increased maize grain yield. This increase in maize yield may be related to increases in yield components (cob length, kernel number per cob, and thousand kernel weight). Although cob number increased significantly with increasing planting density, other yield components decreased with increasing planting density under the same field management [42]. This result is consistent with the previously reported finding that greater kernel number and larger kernels are important parameters for improving grain yield in areas with water and temperature limitations to growth [18].

Additionally, our findings indicated that there was a significant correlation between dry matter accumulation and maize grain yield, but the coefficient of determination was greater for the relationship between dry matter accumulation at physiological maturity and grain yield $\left(2016, R^{2}=\right.$ $\left.0.971 ; 2017, R^{2}=0.975 ; 2018, R^{2}=0.964\right)$ than at silking (2016, $R^{2}=0.770 ; 2017, R^{2}=0.689 ; 2018, R^{2}=0.781$ ) (Fig. S2). Therefore, the effect of dry matter accumulation after silking on maize yield is more important than dry matter accumulation before silking. This finding may be different 
Table 5 Effects of mulching, planting density $\left(D_{L}, 55,000\right.$ and $D_{H}, 90,000$ seeds ha $\left.{ }^{-1}\right)$, and nitrogen fertilizer application rates $\left(N_{0}\right.$, $\mathrm{N}_{120}, \mathrm{~N}_{240}$, and $\mathrm{N}_{360}$; subscript indicates $\mathrm{kg} \mathrm{N} \mathrm{ha}^{-1}$ ) on maize nitrogen assimilation amount after silking (NAAS), nitrogen harvest index (NHI), nitrogen use efficiency (NUE), apparent nitrogen recovery efficiency (NRE), and partial factor productivity of the fertilizer (PFP)

\begin{tabular}{|c|c|c|c|c|c|c|c|c|c|c|c|c|}
\hline \multirow[t]{2}{*}{ Year } & \multirow[t]{2}{*}{ Mulch } & \multirow{2}{*}{$\begin{array}{l}\text { Nitrogen } \\
\left(\mathrm{N}_{\mathrm{kg} / \mathrm{ha}}\right)\end{array}$} & \multicolumn{2}{|c|}{ NAAS $\left(\mathrm{kg} \mathrm{ha}^{-1}\right)$} & \multicolumn{2}{|l|}{$\mathrm{NHI}(\%)$} & \multicolumn{2}{|c|}{ NUE $\left(\mathrm{kg} \mathrm{kg}^{-1}\right)$} & \multicolumn{2}{|l|}{ NRE (\%) } & \multicolumn{2}{|c|}{ PFP $\left(\mathrm{kg} \mathrm{kg}^{-1}\right)$} \\
\hline & & & $D_{L}$ & $D_{H}$ & $D_{L}$ & $D_{H}$ & $D_{L}$ & $D_{H}$ & $D_{L}$ & $D_{H}$ & $D_{L}$ & $\mathrm{D}_{\mathrm{H}}$ \\
\hline \multirow[t]{8}{*}{2015} & No & $N_{0}$ & 29.9 & 31.9 & 64.7 & 54.2 & & & & & & \\
\hline & & $\mathrm{N}_{120}$ & 63.2 & 63.4 & 71.8 & 65.6 & 59.7 & 60.7 & 42.7 & 44.9 & 25.6 & 26.0 \\
\hline & & $\mathrm{N}_{240}$ & 71.5 & 69.5 & 74.9 & 67.4 & 34.2 & 34.3 & 34 & 35 & 20.5 & 20.6 \\
\hline & & $\mathrm{N}_{360}$ & 71.1 & 68.2 & 70.5 & 65.6 & 22.8 & 24.6 & 24.5 & 24.5 & 15.8 & 17.0 \\
\hline & Yes & $\mathrm{N}_{0}$ & 41.6 & 40.9 & 62.9 & 53.6 & & & & & & \\
\hline & & $N_{120}$ & 56.3 & 59.2 & 67.9 & 64.4 & 62.2 & 74.9 & 40.5 & 41 & 26.7 & 32.1 \\
\hline & & $\mathrm{N}_{240}$ & 73.9 & 73.1 & 66.3 & 64.9 & 36.5 & 41.9 & 36.3 & 35.2 & 21.9 & 25.1 \\
\hline & & $N_{360}$ & 67.7 & 68.6 & 65.7 & 61.8 & 24.0 & 29.7 & 25.2 & 25 & 16.6 & 20.5 \\
\hline \multirow[t]{8}{*}{2016} & No & $N_{0}$ & 31.0 & 42.6 & 64.4 & 54.3 & & & & & & \\
\hline & & $\mathrm{N}_{120}$ & 64.3 & 57.3 & 71.5 & 65.4 & 63.3 & 66.0 & 45.8 & 47.2 & 27.1 & 28.3 \\
\hline & & $\mathrm{N}_{240}$ & 72.5 & 74.6 & 74.6 & 67.2 & 36.0 & 37.2 & 36.1 & 37.1 & 21.6 & 22.3 \\
\hline & & $\mathrm{N}_{360}$ & 72.3 & 68.5 & 70.3 & 65.5 & 23.6 & 26.1 & 25.5 & 26.2 & 16.4 & 18.1 \\
\hline & Yes & $\mathrm{N}_{0}$ & 32.9 & 42.4 & 62.7 & 53.7 & & & & & & \\
\hline & & $\mathrm{N}_{120}$ & 64.4 & 60.2 & 67.7 & 64.3 & 66.5 & 80.1 & 43.3 & 44.8 & 28.5 & 34.3 \\
\hline & & $\mathrm{N}_{240}$ & 70.5 & 74.4 & 66.1 & 64.7 & 39.0 & 46.6 & 37.1 & 35.2 & 23.4 & 27.9 \\
\hline & & $N_{360}$ & 69.3 & 69.1 & 65.5 & 61.7 & 25.1 & 31.4 & 26.2 & 25.7 & 17.4 & 21.7 \\
\hline \multirow[t]{8}{*}{2017} & No & $N_{0}$ & 32.6 & 44.3 & 64.2 & 54.4 & & & & & & \\
\hline & & $\mathrm{N}_{120}$ & 65.9 & 59.0 & 71.2 & 65.3 & 68.2 & 70.2 & 42.5 & 43.9 & 29.2 & 30.1 \\
\hline & & $\mathrm{N}_{240}$ & 74.1 & 76.3 & 74.3 & 67.0 & 38.1 & 39.2 & 33.4 & 35.2 & 22.9 & 23.5 \\
\hline & & $\mathrm{N}_{360}$ & 73.9 & 70.1 & 70.1 & 65.4 & 25.7 & 28.6 & 23.2 & 25 & 17.8 & 19.8 \\
\hline & Yes & $\mathrm{N}_{0}$ & 34.5 & 44.0 & 62.6 & 53.8 & & & & & & \\
\hline & & $\mathrm{N}_{120}$ & 66.1 & 61.8 & 67.5 & 64.2 & 69.6 & 84.9 & 41.2 & 41.9 & 29.8 & 36.4 \\
\hline & & $\mathrm{N}_{240}$ & 72.1 & 76.0 & 66.0 & 63.4 & 40.8 & 48.6 & 34.2 & 34.7 & 24.5 & 29.1 \\
\hline & & $\mathrm{N}_{360}$ & 70.9 & 71.1 & 63.8 & 61.2 & 27.0 & 34.7 & 24.3 & 24.5 & 18.7 & 24.0 \\
\hline \multicolumn{13}{|c|}{ Treatment effect ( $P$ values) $)^{a}$} \\
\hline \multicolumn{3}{|c|}{ Year $(Y)$} & \multicolumn{2}{|l|}{$<0.001$} & \multicolumn{2}{|l|}{ ns } & \multicolumn{2}{|l|}{$<0.001$} & \multicolumn{2}{|l|}{ ns } & \multicolumn{2}{|l|}{$<0.001$} \\
\hline \multicolumn{3}{|c|}{ Mulch (M) } & \multicolumn{2}{|l|}{0.002} & \multicolumn{2}{|l|}{$<0.001$} & \multicolumn{2}{|l|}{$<0.001$} & $<0.001$ & & $<0.001$ & \\
\hline Nitrog & & & $<0.001$ & & $<0.001$ & & $<0.001$ & & $<0.001$ & & $<0.001$ & \\
\hline Densit & & & ns & & $<0.001$ & & $<0.001$ & & $<0.001$ & & $<0.001$ & \\
\hline$Y * M$ & & & ns & & ns & & ns & & ns & & ns & \\
\hline$Y * N$ & & & ns & & ns & & $<0.001$ & & ns & & 0.011 & \\
\hline$Y * D$ & & & ns & & ns & & 0.039 & & ns & & 0.012 & \\
\hline$M * N$ & & & $<0.001$ & & $<0.001$ & & $<0.001$ & & $<0.001$ & & $<0.001$ & \\
\hline$M * D$ & & & ns & & $<0.001$ & & $<0.001$ & & 0.01 & & $<0.001$ & \\
\hline$N * D$ & & & ns & & $<0.001$ & & $<0.001$ & & 0.02 & & 0.004 & \\
\hline$Y * M$ & & & ns & & ns & & ns & & ns & & ns & \\
\hline$Y * M$ & & & ns & & ns & & ns & & ns & & ns & \\
\hline$Y * N$ & & & ns & & ns & & ns & & ns & & ns & \\
\hline$M * N$ & & & ns & & $<0.001$ & & $<0.001$ & & ns & & 0.004 & \\
\hline$Y^{*} M$ & * $D$ & & ns & & ns & & ns & & ns & & ns & \\
\hline
\end{tabular}

${ }^{a}$ ns Not significant $(p>0.05)$ 
from some previous studies that demonstrated that the period from jointing to silking is the most sensitive period for water and nutrient requirements [43, 44]. This may be due to our focus on maize grain yield (a result) while previous studies focused on maize growth (a process). Part of the reason for this phenomenon is that dry matter accumulation and $\mathrm{N}$ translocation play an important role between silking and physiological maturity. The source of dry matter and $\mathrm{N}$ translocation from organs to grain is the dry matter accumulation that occurs between jointing to silking [45-47].

While the benefits of plastic mulch have been well documented in previous studies [48-50], the present study showed that the effect of mulch on maize grain yield varied with planting density and $\mathrm{N}$ fertilizer application rate (Fig. 3). In three consecutive growing seasons, under low planting density, plastic mulch increased maize grain yield by $5.5 \%$ when averaged across all other factors, while under the high planting density, plastic mulch increased maize grain yield by $20.8 \%$ when averaged across all other factors. The value of mulch for increasing maize grain yield increased with increasing $\mathrm{N}$ fertilizer application rate under the high planting density. This result is consistent with the hypothesis that plastic mulch used in conjunction with high planting density and high $\mathrm{N}$ fertilizer application rate can increase maize grain yield.

In addition, the present study demonstrated that plastic mulch improved maize grain yield as planting density and $\mathrm{N}$ fertilizer amount increased. However, under high planting density, decreased soil evaporation is offset by increased maize transpiration [51], with the result that planting density has no significant effect on water consumption and soil water storage in arid and semi-arid regions $[52,53]$. Therefore, it can be inferred that $\mathrm{N}$ uptake and $\mathrm{N}$ translocation from maize vegetative organs significantly affects maize yield under the combined action of mulch, $\mathrm{N}$ fertilizer application rate, and planting density.

\section{Maize nitrogen uptake, translocated nitrogen from vegetative organs and translocated nitrogen efficiency responses to mulch, planting density, and nitrogen fertilizer application rate}

Grain $\mathrm{N}$ uptake at physiological maturity can be divided into two parts: $1 . \mathrm{N}$ assimilation from the soil, and $2 . \mathrm{N}$ translocated from vegetative organs [54-56]. The present study showed that mulch, planting density, and $\mathrm{N}$ fertilizer application rate significantly affected $\mathrm{N}$ uptake at silking and at physiological maturity in different plant organs, and also that there was a significant three-way interaction effect among those three factors on $\mathrm{N}$ uptake of different organs, except leaf $\mathrm{N}$ uptake at silking (Table 3). Previous studies have noted a high correlation between $\mathrm{N}$ uptake and crop $\mathrm{N}$ demand $[57,58]$, and a strong correlation between plant $\mathrm{N}$ uptake and dry matter accumulation [59]. The present study showed that there was a strong correlation between maize grain yield and $\mathrm{N}$ uptake (Fig. S3). This result is consistent with the results of [60] who showed that the more nitrogen absorbed and accumulated in the whole plant, the greater contribution to grain yield. The correlation coefficient between $\mathrm{N}$ uptake and maize grain yield varied with different vegetative organs, with higher coefficients of determination observed for leaf (Fig. S4) and stem (Fig. S5) than for cob (Fig. S6). This result suggests that maize genotype selection for increased $\mathrm{N}$ uptake in leaves and stems is an effective way to improve total $\mathrm{N}$ uptake.

Plastic mulch increases soil moisture and $\mathrm{N}$ uptake, thus increasing maize grain yield [61, 62]. In our study, plastic mulch increased total plant $\mathrm{N}$ uptake by $13 \%$ at silking and by $8.2 \%$ at physiological maturity compared with no mulch when averaged across all factors. Plastic mulch reduces evaporation losses [63], and the resultant higher soil moisture helps reduce ammonia volatilization and enhances nitrate uptake by plants [64, 65]. Moreover, converting ammonia to nitrates helps reduce reactive nitrogen emissions and maintains soil nitrogen content $[7,66]$. This study demonstrated that plant $\mathrm{N}$ uptake under high planting density was only 3.5 and $3.4 \%$ higher than under low planting density when averaged across all factors at silking and physiological maturity, respectively. We therefore conclude that plant $\mathrm{N}$ uptake was more sensitive to mulch than to planting density. There may be two reasons for why high planting density increased plant $\mathrm{N}$ uptake. One reason may be increased utilization rate of light energy, and the other reason may be increased assimilation of soil $\mathrm{N}$ [67-69]. That is, the effect of promoting plant $\mathrm{N}$ uptake by increasing the moisture and temperature conditions of the soil with plastic mulch was greater than that of improving light utilization efficiency with increased planting density.

By comparing $\mathrm{N}$ uptake with $\mathrm{N}$ translocated, it is possible to infer that $\mathrm{N}$ uptake and $\mathrm{N}$ translocated generally balanced each other (i.e., higher $\mathrm{N}$ uptake corresponded to lower $\mathrm{N}$ translocated with no mulch and lower planting population density, Fig. 4 vs Fig. 6). With the mulch treatment, there was a clear increase in both plant $\mathrm{N}$ uptake and $\mathrm{N}$ translocated as $\mathrm{N}$ fertilizer application rate increased. This means that a higher proportion of plant $\mathrm{N}$ was retained in the vegetative organs at maturity, thereby reducing $\mathrm{N}$ translocation efficiency under no mulch and low planting density. Therefore, it can be concluded that the most effective way to improve NUE is to improve plant $\mathrm{N}$ uptake and $\mathrm{N}$ translocated. Previous studies have demonstrated that $\mathrm{N}$ translocation and accumulation are equally important to grain yield [ 55 , 
70]. In the present study, under the mulch treatment, plant $\mathrm{N}$ uptake increased with increasing $\mathrm{N}$ fertilizer application rate at silking (Fig. 4), which promoted $\mathrm{N}$ transfer from maize vegetative organs to kernels (Fig. 6). Together, the $\mathrm{N}$ uptake and $\mathrm{N}$ translocation increased grain $\mathrm{N}$ uptake at physiological maturity and improved thousand kernel weight (Table 2), ultimately resulting in high maize grain yield.

In this study, we examined the impact of mulch, planting density, and $\mathrm{N}$ fertilizer application rate on $\mathrm{N}$ translocation efficiency in maize, and found that there was a significant three-way interaction effect among the three factors on $\mathrm{N}$ translocation efficiency (Table 4). $\mathrm{N}$ translocation efficiency showed significant correlation with maize grain yield and plant $\mathrm{N}$ uptake (Fig. S7). However, these results did not follow the pattern of increased $\mathrm{N}$ translocation efficiency with increasing $\mathrm{N}$ fertilizer application rate (Fig. 7). Especially in the case of the no mulch treatment, the $\mathrm{N}$ translocation efficiency first increased and then decreased with increasing $\mathrm{N}$ fertilizer application rate. These results negate our hypothesis that using mulch in conjunction with high planting density can increase $\mathrm{N}$ translocation efficiency with increasing $\mathrm{N}$ fertilizer application rate. This may be due to the limitation of $\mathrm{N}$ uptake and storage in single maize plants. Although the $\mathrm{N}$ translocation efficiency did not follow the pattern of increasing with increasing $\mathrm{N}$ fertilizer application rate, total $\mathrm{N}$ uptake by maize was positively correlated with $\mathrm{N}$ fertilizer application rate. Adequate soil moisture and nutrients are beneficial to the growth and development of maize, which directly affect the accumulation and transport of $\mathrm{N}$, and thus the total $\mathrm{N}$ uptake by maize [71, 72]. Previous studies have shown that increasing $\mathrm{N}$ fertilizer application rate can increase the $\mathrm{N}$ uptake before silking, and further improve $\mathrm{N}$ translocation from leaves and stems to grain by improving the absorption of water and nutrients by roots under better soil hydrothermal conditions (mulch vs. no mulch) [7377]. Therefore, combining trait selection with $\mathrm{N}$ management research to improve $\mathrm{N}$ translocation efficiency is an effective way to improve NUE.

\section{Nitrogen harvest index and nitrogen use efficiency responses to mulch, planting density, and nitrogen fertilizer application rate}

Nitrogen assimilation amount after silking (NAAS) plays an important role in increasing grain yield and reducing $\mathrm{N}$ residue in soil [78-81]. The present study showed that NAAS initially increased and then decreased with increasing $\mathrm{N}$ fertilizer application rate, and that when averaged across all factors, the highest value of NAAS was obtained with the $\mathrm{N}_{240}$ application rate. This result may be related to soil $\mathrm{N}$ availability being dependent on the physiological ability of roots to take up and assimilate $\mathrm{N}$
[34]. In addition, mineralized $\mathrm{N}$ can provide at most 50\% of the $\mathrm{N}$ absorbed by maize during a single growing season despite extensive application of $\mathrm{N}$ fertilizer [82]. Planting density had no significant interaction effect on NAAS, but mulch, planting density, and $\mathrm{N}$ fertilizer application had a significant interaction effect on nitrogen harvest index (NHI), nitrogen use efficiency (NUE), and partial factor productivity of the fertilizer (PFP). This phenomenon may be related to the limit storing capacity of $\mathrm{N}$ absorbing. Vegetative organs are transformed from sink organs to source organs, and this change is accompanied by $\mathrm{N}$ fluxes from old tissues to young tissues and reproductive organs prior to physiological maturity [83].

During the three consecutive growing seasons of this study, the coefficient of determination for the relationship between NASS and grain yield was higher than the coefficient of determination for the relationship between $\mathrm{NHI}$ and grain yield (Fig. S8). It has been observed that an increase in NUE is associated with an increase in $\mathrm{NHI}$, which in turn was associated with a higher $\mathrm{N}$ translocation efficiency in later stages of the grain filling period [84, 85]. The results of our study demonstrate that NHI and $\mathrm{N}$ translocation efficiency have a significant linear correlation $\left(2016, \mathrm{R}^{2}=0.814, p<0.01 ; 2017\right.$, $\mathrm{R}^{2}=0.811, p<0.01 ; 2018, \mathrm{R}^{2}=0.809, p<0.01$ ) (Fig. S9). In our experiment, the higher NHI of the $\mathrm{N}_{240}$ treatment was associated to higher $\mathrm{N}$ translocation efficiency. These results partially agree with increasing planting density, and reducing $\mathrm{N}$ fertilizer application rate can significantly promote $\mathrm{N}$ absorption, such that high total $\mathrm{N}$ accumulation and absorption of solar radiation can result in high grain yield and NHI $[42,86]$.

There was a negative correlation between the $\mathrm{N}$ fertilizer application rate and the NUE. Reducing $\mathrm{N}$ fertilizer application rate can balance $\mathrm{N}$ supply with crop demand for $\mathrm{N}$ [87]. Our study also showed that the combination of mulch, high planting population density, and high $\mathrm{N}$ fertilizer application rate $\left(\mathrm{N}_{360}\right)$ resulted in the highest total $\mathrm{N}$ uptake, but the lowest NUE. Increasing planting density can increase NUE, no matter how much $\mathrm{N}$ fertilizer is applied, and NUE will increase significantly under mulch treatment $[88,89]$. This study showed that although NUE at $\mathrm{N}_{240}$ was lower than NUE at $\mathrm{N}_{120}$, the interaction effect between planting density and mulch was significant, which effectively improved NUE.

In order to better understand the effects of mulch, planting density, and $\mathrm{N}$ fertilizer application rate on crop $\mathrm{N}$ dynamics, NRE was calculated in this study. It has been reported that the relative importance of NRE in determining NUE varies with $\mathrm{N}$ fertilizer application rate [37]. The NRE is more representative of the $\mathrm{N}$ uptake efficiency of crops [90]. In our experiment, NRE and NUE were the same, and the highest values of both occurred at the lowest $\mathrm{N}$ fertilizer application rate $\left(\mathrm{N}_{120}\right)$. 
However, NRE and NUE exhibited different responses to $\mathrm{N}$ fertilizer application rate for different planting densities and mulch treatments $[25,91]$. There was a significant linear correlation between NRE and NUE and between PFP and NUE (Fig. S10). In addition, our study indicated that mulch was an effective measure to improve NRE with $\mathrm{N}$ application. One reason might be related to the fact that mulching increases the amount of soil heat available for maize growth during early growth stages, which has a significant impact on crop $\mathrm{N}$ uptake and $\mathrm{N}$ accumulation [92, 93]. Another reason might be a large amount of $\mathrm{N}$ was accumulated in maize vegetative organs under the mulch treatment at silking, which significantly increased $\mathrm{N}$ translocation from vegetative organs to reproductive organs during later growth stages [94]. There is no doubt that PFP decreases with increasing fertilizer application rate, and PFP was higher in mulch than with no mulch in high and low planting densities. The NUE was higher with high planting density than with low planting density under different mulch treatments and $\mathrm{N}$ fertilizer application rates, but NRE under high planting density was higher than under low planting density only under the no mulch treatment. Thus, the combination of increasing planting density and reducing $\mathrm{N}$ fertilizer application rate with mulch can better improve resource utilization by maize.

\section{Conclusions}

In conclusion, mulch, planting density, and $\mathrm{N}$ fertilizer application rate not only have significant effects on improving maize grain yield and NUE, but also on $\mathrm{N}$ uptake, $\mathrm{N}$ translocation, and $\mathrm{N}$ translocation efficiency. Our results showed clearly that under high planting density, the combination of mulch and moderate $\mathrm{N}$ fertilizer application rate was the optimal strategy for increasing maize grain yield. Increasing planting density can promote $\mathrm{N}$ uptake and increase cob number, which results in higher grain yield and NUE at the same $\mathrm{N}$ fertilizer application rate. However, $\mathrm{N}$ translocation and $\mathrm{N}$ translocation efficiency did not increase with increasing $\mathrm{N}$ fertilizer application rate and planting density. We infer from this that approaches to improve maize grain yield and NUE should focus on a combination of field management and genetic manipulation to improve inorganic $\mathrm{N}$ uptake and allocation, and $\mathrm{N}$ translocation and its regulation. Due to excessive input of $\mathrm{N}$ fertilizer, the NUE of $\mathrm{N}_{360}$ was low. At the same time, there was no significant difference in maize grain yield when $\mathrm{N}$ fertilizer application rate reached a level higher than $\mathrm{N}_{240}$. In order to obtain relatively high grain yield and high NUE at the same time, the commonly used $\mathrm{N}$ fertilizer application rate $\left(\mathrm{N}_{360}\right)$ should be reduced. Our research may be valuable for the determining environmentally friendly methods for improving $\mathrm{N}$ translocation to achieve efficient use of $\mathrm{N}$ fertilizer.

\section{Methods \\ Plant materials and growth conditions}

Seeds of maize (cv. 'Zhengdan 958') were obtained from the Institute of Grain Production, Henan Academy of Agricultural Sciences. All materials were grown in the field in accordance with the local legislation. The high dominance hybrid Zhengdan 958 is planted all over China. Its parental inbreeding belonged to two different heterosis groups: Zheng 58 belonged to the PA heterosis group, a subgroup of stiff stalk, and Chang 7-2 belonged to the TSPT heterosis group, a subgroup of non-stiff stalk. The field experiment was carried out during the 2016, 2017, and 2018 maize growing seasons on farms in the Northwest Loess Plateau (36 $39^{\prime} \mathrm{N}, 109^{\circ} 11^{\prime}$ E, altitude $1109 \mathrm{~m}$ above sea level) in Ansai District, Shaanxi Province, China. The climate at the planting site is highland continental monsoon. The average annual temperature is $8.7^{\circ} \mathrm{C}$ and the mean annual rainfall is $511 \mathrm{~mm}$, with about $70 \%$ of the annual rainfall occurring in the maize growing season [95]. Total rainfall at the site was measured using a rainfall recorder (wi92859, Dongxi Instrument Technology Ltd., Beijing, China). Total annual precipitation was 382, 499, and $578 \mathrm{~mm}$ in 2016, 2017, and 2018, respectively, and the total rainfall during the maize growing season was 256,460 , and 455 $\mathrm{mm}$. The average sand, silt, and clay contents in the 0 $80 \mathrm{~cm}$ soil profile were $15.9,66.7$, and $17.4 \%$, respectively. Bulk density was measured by the core method, using cores that measured $3 \mathrm{~cm}$ in diameter, $10 \mathrm{~cm}$ in length, and $70.68 \mathrm{~cm}^{3}$ in volume. Field capacity at $33 \mathrm{kPa}$ was determined using a pressure-membrane extraction apparatus. Soil organic matter was determined using the Walkley-Black method. The available phosphorus was determined using the Olsen method. Available potassium was determined by $\mathrm{H}_{2} \mathrm{SO}_{4}-\mathrm{HCLO}_{4}$ digestion and the molybdenum antimony-ascorbic acid colorimetric method. The soil properties of the top $80 \mathrm{~cm}$ were as follows: $\mathrm{pH}$ (1:2.5 soil: water), 8.5 ; bulk density, $1.28 \mathrm{~g}$ $\mathrm{cm}^{-3}$; organic matter, $15.2 \mathrm{~g} \mathrm{~kg}^{-1}$; total $\mathrm{N}, 0.77 \mathrm{~g} \mathrm{~kg}^{-1}$; available phosphorus, $33.2 \mathrm{mg} \mathrm{kg}^{-1}$; available potassium, $138.2 \mathrm{mg} \mathrm{kg}^{-1}$; and mineral $\mathrm{N}, 13.1 \mathrm{mg} \mathrm{kg}^{-1}$.

\section{Experimental design}

The experiment was arranged in a randomized complete block design with three factors $(2$ mulch levels $\times 2$ planting densities $\times 4 \mathrm{~N}$ fertilizer application rates) replicated four times. The two mulch levels were plastic mulch and no-mulch. Two planting densities typical for this area were used in this study (low planting density of 55,000 seeds $\mathrm{ha}^{-1}$ and high planting density of 90,000 seeds $\mathrm{ha}^{-1}$ ). The four $\mathrm{N}$ fertilizer application rates were 0 
$\left(\mathrm{N}_{0}\right), 120\left(\mathrm{~N}_{120}\right), 240\left(\mathrm{~N}_{240}\right)$, and $360\left(\mathrm{~N}_{360}\right) \mathrm{kg} \mathrm{N} \mathrm{ha}^{-1}$ applied as urea $(46.4 \% \mathrm{~N})$. The $\mathrm{N}_{360}$ rate is the rate commonly applied by local farmers). The plots were selected randomly with four replicates. Individual plots were 46 $\mathrm{m}^{2}(4.6 \mathrm{~m} \times 10 \mathrm{~m})$ and each plot was separated by a 20 $\mathrm{cm}$-wide ridge as a barrier. Before mulching, each treatment received half of the total amount of $\mathrm{N}$ fertilizer as a basal $\mathrm{N}$ fertilizer application $\left(\mathrm{N}_{0}, 0 \mathrm{~kg} \mathrm{ha}^{-1} ; \mathrm{N}_{120}, 60\right.$ $\mathrm{kg} \mathrm{ha}^{-1} ; \mathrm{N}_{240}, 120 \mathrm{~kg} \mathrm{ha}^{-1} ; \mathrm{N}_{360}, 180 \mathrm{~kg} \mathrm{ha}^{-1}$ ) as well as $80 \mathrm{~kg} \mathrm{ha}^{-1}$ of phosphorus pentoxide $\left(\mathrm{P}_{2} \mathrm{O}_{5} 44 \%\right)$ and 80 $\mathrm{kg} \mathrm{ha}^{-1}$ of potassium oxide $\left(\mathrm{K}_{2} \mathrm{O} 60 \%\right)$, which were applied at the same time. The other half of the $\mathrm{N}$ fertilizer application was applied in early July each year as topdressing. For top-dressed N, N fertilizer was band applied in the middle of the furrow rows at a depth of 5 $\mathrm{cm}$. All plots were prepared in a ridge-furrow pattern with alternating narrow space between rows $(15-\mathrm{cm}$ high $\times 40-\mathrm{cm}$ wide) and wide space between rows $(10-\mathrm{cm}$ high $\times 70-\mathrm{cm}$ wide). Colorless, transparent, $0.008-\mathrm{mm}$ thick polyethylene film plastic mulch was purchased from the local farmers' market, and the entire ridge surface was covered after plot preparation. Technique of the alternately planting of wide $(70-\mathrm{cm})$ and narrow $(40-$ $\mathrm{cm})$ row spacing was used with plant spacing of $35 \mathrm{~cm}$ $\left(55,000\right.$ seeds $\left.\mathrm{ha}^{-1}\right)$ and $20 \mathrm{~cm}$ (90,000 seeds ha $\left.{ }^{-1}\right)$. Maize (cv. 'Zhengdan 958') was planted on 23 April 2016, 20 April 2017, and 25 April 2018, and harvested on 12 October 2016, 8 October 2017, and 13 October 2018. After harvest, the plastic film was gathered and recycled by the manufacturer, and the soil was then plowed to a depth of $25 \mathrm{~cm}$. There was no irrigation throughout the growth period.

\section{Plant sampling and analysis}

Four adjacent plants in a row of each plot were randomly harvested at the jointing stage (JS, 7-leaf stage), tasseling stage (TS, 14-leaf stage), silking stage (SS, silks visible outside the husks), and physiological maturity stage (PMS, approximately 50 days after filling stage). The sampled plants were cut off at ground level and separated into stems (including sheaths, stems, and bract), leaves, cobs, and grains. All the plants in two randomlyselected adjacent rows of maize (rows sampled during maize silking stage are excluded) in each plot were cut off at ground level, the number of cobs counted before all the parts were oven-dried at $75^{\circ} \mathrm{C}$ to constant weight. Ten ears in each plot were randomly sampled for the determination of yield components: cob number, kernel number per cob and 1000-kernel weight [96]. Then the dried samples were weighed to determine dry matter accumulation (DMA) and ground to a fine powder for $\mathrm{N}$ measurement. The total $\mathrm{N}$ concentration in each plant organ was determined using the Kjeldahl method [56].

\section{Calculations}

The different $\mathrm{N}$ uptake, $\mathrm{N}$ translocation, and $\mathrm{N}$ translocation efficiency parameters were calculated following methods described by $[45,75,97]$. The plant $\mathrm{N}$ uptake was calculated as:

$$
P N U=\frac{D M A \times N C}{1000}
$$

where $P N U$ is the plant total nitrogen uptake $(\mathrm{kg} \mathrm{N}$ $\left.\mathrm{ha}^{-1}\right), D M A$ is the plant dry matter accumulation $(\mathrm{kg}$ $\left.\mathrm{ha}^{-1}\right)$, and $N C$ is the nitrogen concentration $\left(\mathrm{mg} \mathrm{g}^{-1}\right)$.

$$
N T=P N U_{S S}-P N U_{P M S}
$$

where $N T$ is the nitrogen translocation $\left(\mathrm{kg} \mathrm{Nha}^{-1}\right)$, $P N U_{S S}$ is the plant total nitrogen uptake of vegetative organs at silking $\left(\mathrm{kg} \mathrm{Nha}^{-1}\right)$, and $P N U_{P M S}$ is the plant total nitrogen uptake of vegetative organs at physiological maturity $\left(\mathrm{kg} \mathrm{N} \mathrm{ha}^{-1}\right)$.

$$
N T E=\frac{N T}{P N U_{S S}} \times 100
$$

where NTE is the nitrogen translocation efficiency (\%), $N T$ is the nitrogen translocation $\left(\mathrm{kg} \mathrm{Nha}^{-1}\right)$, and $P N U_{S S}$ is the plant total nitrogen uptake of vegetative organs at silking $\left(\mathrm{kg} \mathrm{Nha}^{-1}\right)$.

$$
L N T=L N U_{S S}-L N U_{P M S}
$$

where $L N T$ is the leaf nitrogen translocation $\left(\mathrm{kg} \mathrm{N} \mathrm{ha}^{-1}\right)$, $L N U_{S S}$ is the leaf total nitrogen uptake at silking $(\mathrm{kg} \mathrm{N}$ $\mathrm{ha}^{-1}$ ), and $L N U_{P M S}$ is the leaf total nitrogen uptake at physiological maturity $\left(\mathrm{kg} \mathrm{N} \mathrm{ha}^{-1}\right)$.

$$
S N T=S N U_{S S}-S N U_{P M S}
$$

where $S N T$ is the stem nitrogen translocation $(\mathrm{kg} \mathrm{N}$ $\left.\mathrm{ha}^{-1}\right), S N U_{S S}$ is the stem total nitrogen uptake at silking $\left(\mathrm{kg} \mathrm{Nha}^{-1}\right)$, and $S N U_{P M S}$ is the stem total nitrogen uptake at physiological maturity $\left(\mathrm{kg} \mathrm{N} \mathrm{ha}^{-1}\right)$.

$$
E N T=E N U_{S S}-E N U_{P M S}
$$

where ENT is the cob nitrogen translocation $(\mathrm{kg} \mathrm{N}$ $\left.\mathrm{ha}^{-1}\right), E N U_{S S}$ is the cob total nitrogen uptake at silking $\left(\mathrm{kg} \mathrm{Nha}^{-1}\right)$, and $E N U_{P M S}$ is the cob total nitrogen uptake at physiological maturity $\left(\mathrm{kg} \mathrm{N} \mathrm{ha}^{-1}\right)$.

$$
L N T E=\frac{L N T}{P N U_{S S}} \times 100
$$

where $L N T E$ is the leaf nitrogen translocation efficiency (\%), LNT is the leaf nitrogen translocation $\left(\mathrm{kg} \mathrm{N} \mathrm{ha}^{-1}\right)$, and $P N U_{S S}$ is the plant total nitrogen uptake at silking $\left(\mathrm{kg} \mathrm{Nha}^{-1}\right)$.

$$
S N T E=\frac{S N T}{P N U_{S S}} \times 100
$$


where SNTE is the stem nitrogen translocation efficiency (\%), SNT is the stem nitrogen translocation $\left(\mathrm{kg} \mathrm{N} \mathrm{ha}^{-1}\right)$, and $P N U_{S S}$ is the plant total nitrogen uptake at silking $\left(\mathrm{kg} \mathrm{Nha}^{-1}\right)$.

$$
E N T E=\frac{E N T}{P N U_{S S}} \times 100
$$

where ENTE is the cob nitrogen translocation efficiency (\%), ENT is the cob nitrogen translocation $\left(\mathrm{kg} \mathrm{N} \mathrm{ha}^{-1}\right)$ and $P N U_{S S}$ is the plant total nitrogen uptake at silking $\left(\mathrm{kg} \mathrm{Nha}^{-1}\right)$.

$$
N A A S=G N U_{P M S}-T N T
$$

where NAAS is the nitrogen assimilation amount after silking $\left(\mathrm{kg} \mathrm{N} \mathrm{ha}^{-1}\right), G N U_{P M S}$ is the grain nitrogen uptake of vegetative organs at physiological maturity $(\mathrm{kg} \mathrm{N}$ $\mathrm{ha}^{-1}$ ), and TNT is the sum of leaves, stems, and cob nitrogen translocation $\left(\mathrm{kg} \mathrm{N} \mathrm{ha}^{-1}\right)$.

$$
N H I=\frac{G N U}{P N U-A} \times 100
$$

where $N H I$ is the nitrogen harvest index (\%), GNU is the grain total nitrogen uptake at physiological maturity $(\mathrm{kg}$ $\mathrm{Nha}^{-1}$ ), and PNU-A is the total nitrogen uptake by aboveground organs at physiological maturity $(\mathrm{kg} \mathrm{N}$ $\mathrm{ha}^{-1}$ ).

$$
N U E=\frac{G Y}{N_{\text {amount }}}
$$

where NUE is the nitrogen use efficiency $\left(\mathrm{kg} \mathrm{kg}^{-1}\right), G Y$ is the grain yield $\left(\mathrm{kg} \mathrm{ha}^{-1}\right)$, and $N_{\text {amount }}$ is the nitrogen fertilizer application amount $\left(\mathrm{kg} \mathrm{N} \mathrm{ha}^{-1}\right)$.

$$
N R E=\frac{P N U-P M S_{F}-P N U-P M S_{Z}}{N_{\text {amount }}} \times 100
$$

where $N R E$ is the nitrogen apparent recovery efficiency (\%), $P N U-P M S_{F}$ is the plant total nitrogen uptake in the nitrogen fertilizer application plot at physiological maturity $\left(\mathrm{kg} \mathrm{Nha}^{-1}\right)$, and $P N U-P M S_{Z}$ is the plant total nitrogen uptake in the no fertilization plot at physiological maturity $\left(\mathrm{kg} \mathrm{ha}^{-1}\right)$.

$$
P F P=\frac{G Y}{N_{\text {amount }}+P_{\text {amount }}+K_{\text {amount }}}
$$

where PFP is the partial factor productivity of the fertilizer $\left(\mathrm{kg} \mathrm{ha}^{-1}\right), G Y$ is the grain yield $\left(\mathrm{kg} \mathrm{ha}^{-1}\right)$, $N_{\text {amount }}$ is the amount of nitrogen fertilizer application $\left(\mathrm{kg} \mathrm{N} \mathrm{ha}^{-1}\right), P_{\text {amount }}$ is the amount of phosphate fertilizer application $\left(\mathrm{kg} \mathrm{P}_{2} \mathrm{O}_{5} \mathrm{ha}^{-1}\right)$, and $K_{\text {amount }}$ is the amount of potassium fertilizer application $\left(\mathrm{kg} \mathrm{K}_{2} \mathrm{O} \mathrm{ha}^{-1}\right)$.

\section{Statistical analysis}

Three-way analysis of variance (ANOVA), with $\mathrm{N}$ application rates, plastic mulch, and planting density as the three fixed factors, was used to assess variations in each indicator. Differences between all treatments were detected using least significant difference (LSD) testing at the 0.05 significance level. We compared each indicator by use of one-way ANOVA, followed by post-hoc pairwise comparisons (Tukey's Honestly Significant Difference $[\mathrm{HSD}]$ procedure), if warranted. Statistical analyses and data plotting were performed using SPSS Statistics Software 16.0 (https://www.ibm.com/products/spss-statistics) and Sigma Plot $14.0 \mathrm{https}: / /$ systatsoftware.com/ products/sigmaplot/), respectively.

\section{Supplementary information}

Supplementary information accompanies this paper at https://doi.org/10 1186/s12870-020-02477-2.

Additional file 1: Figure $\mathbf{S} 1$. The relationship between nitrogen fertilizer application rate and maize grain yield in low plant population density and high plant population density in 2016, 2017, and 2018. Figure S2. The relationship between aboveground dry matter accumulation and maize grain yield in 2016, 2017, and 2018. Figure S3. The relationship between plant total nitrogen uptake and maize grain yield 2016, 2017, and 2018. Figure S4. The relationship between leaf nitrogen uptake and maize grain yield in 2016, 2017, and 2018. Figure S5. The relationship between stem nitrogen uptake and maize grain yield in 2016, 2017, and 2018. Figure S6. The relationship between ear axis nitrogen uptake and maize grain yield in 2016, 2017, and 2018.

Figure S7. The relationship between translocated nitrogen efficiency and grain yield and translocated nitrogen efficiency and plant nitrogen uptake in 2016, 2017, and 2018. Figure S8. The relationship between nitrogen assimilation amount after silking stage and grain yield and nitrogen harvest index and grain yield in 2016, 2017, and 2018. Figure S9. The relationship between translocated nitrogen and nitrogen harvest index and translocated nitrogen efficiency and nitrogen harvest index in 2016, 2017, and 2018. Figure S10. The relationship between nitrogen use efficiency and apparent nitrogen use efficiency and nitrogen use efficiency and partial factor productivity of the fertilizer in 2016, 2017, and 2018.

\section{Abbreviations}

N: Nitrogen; NUE: Nitrogen use efficiency; PA: Group A germplasm derived from modern U.S. hybrids; TSPT: Tangsipingtou; JS: Jointing stage; TS: Tasseling stage; SS: Silking stage; PMS: Physiological maturity stage; DMA: Dry matter accumulation; PNU: Plant total nitrogen uptake;

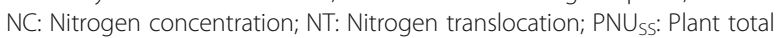
nitrogen uptake of vegetative organs at silking; $\mathrm{PNU}_{\mathrm{PM} \text { : }}$ : Plant total nitrogen uptake of vegetative organs at physiological maturity; NTE: Nitrogen translocation efficiency; LNT: Leaf nitrogen translocation; LNU $U_{s s}$ : Leaf total nitrogen uptake at silking; LNU PMs: Leaf total nitrogen uptake at physiological maturity; SNT: Stem nitrogen translocation; SNU ss: $_{\text {Stem total }}$ nitrogen uptake at silking; SNU PMs: Stem total nitrogen uptake at

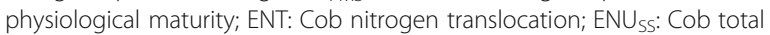

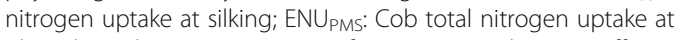
physiological maturity; LNTE: Leaf nitrogen translocation efficiency; ENTE: Cob nitrogen translocation efficiency; NAAS: Nitrogen assimilation

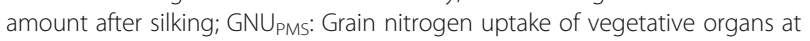
physiological maturity; TNT: Sum of leaves, stems, and cob nitrogen translocation; NHI: Nitrogen harvest index; GNU: Grain total nitrogen uptake at physiological maturity; PNU-A: Total nitrogen uptake by aboveground organs at physiological maturity; GY: Grain yield; $\mathrm{N}_{\text {amount: }}$ Nitrogen fertilizer application amount; NRE: Nitrogen apparent recovery efficiency; PNU$\mathrm{PMS}_{\mathrm{F}}$ : Plant total nitrogen uptake in the nitrogen fertilizer application plot at 
physiological maturity; PNU-PMS: Plant total nitrogen uptake in the no fertilization plot at physiological maturity; PFP: Partial factor productivity of the fertilizer; $P_{\text {amount }}$ : Amount of phosphate fertilizer application; $K_{\text {amount }}$ : Amount of potassium fertilizer application; ANOVA: Analysis of variance; LSD: Least significant difference; HSD: Honestly significant difference

\section{Acknowledgements}

We thank Mr. Meng and Mr. Xing for providing us with experimental field plots and helping us to collect the data. We are very grateful to Dr. David Nielsen for helping us edit the English language.

\section{Authors' contributions}

XW and YX conceived and designed research; GW, ML and TG conducted experiments; ML and TG collected and analyzed the data. XW wrote the manuscript; NCT and YX provided valuable suggestions on the manuscript; $X W$ and $Y X$ revised the manuscript; $X W$ obtained funding and is responsible for this article. All authors read and approved the manuscript.

\section{Funding}

This research was supported by the National Natural Science Foundation of China $(51809224,51669034)$ and the National Key Research and Development Program of China (grant no. 2017YFC0504704), and Shaanxi Innovation Talent Promotion Program - Young Science and Technology Star Project (2018KJXX080). The funding bodies had no role in study design, data collection, analysis and interpretation, decision to public, or writing of the manuscript.

\section{Availability of data and materials}

Datasets used in the current study are available from the corresponding author on reasonable request.

\section{Ethics approval and consent to participate} Not applicable.

\section{Consent for publication}

Not applicable.

\section{Competing interests}

The authors declare that they have no competing interests.

\section{Author details}

'College of Life Sciences, Yan'an University, Yan'an 716000, Shaanxi, China. ${ }^{2}$ The UWA Institute of Agriculture and UWA School of Agriculture and Environment, The University of Western Australia, M082, Locked Bag 5005, Perth, WA 6001, Australia.

Received: 3 December 2019 Accepted: 1 June 2020

Published online: 19 June 2020

\section{References}

1. Cassman KG. Ecological intensification of cereal production systems: Yield potential, soil quality, and precision agriculture. Proc Natl Acad Sci. 1999; 96(11):5952-9.

2. Tong $\mathrm{C}$, Hall CAS, Wang $\mathrm{H}$. Land use change in rice, wheat and maize production in China (1961-1998). Agric Ecosyst Environ. 2003;95(2):523-36.

3. Deng $X$, Shan L, Zhang H, Turner NC. Improving agricultural water use efficiency in arid and semiarid areas of China. Agric Water Manag. 2006; 80(1-3):23-40

4. Wang X, Wang G, Guo T, Xing Y, Mo F, Wang H, Fan J, Zhang F. Effects of plastic mulch and nitrogen fertilizer on the soil microbial community, enzymatic activity and yield performance in a dryland maize cropping system. Eur J Soil Sci. 2020;1:1-13.

5. Fan J, Wang X, Zhang F, Ma X, Wu L. Predicting daily diffuse horizontal solar radiation in various climatic regions of China using support vector machine and tree-based soft computing models with local and extrinsic climatic data. J Clean. Prod. 2020;248:119264.

6. Torres S, Moran E, Silva R. Property rights and the soybean revolution: Shaping how China and Brazil are telecoupled. Sustainability. 2017:9(6):954

7. Ladha JK, Pathak H, J Krupnik T, Six J, van Kessel C. Efficiency of fertilizer nitrogen in cereal production: retrospects and prospects. In: Advances in Agronomy, vol. 87. Cambridge: Academic Press; 2005. p. 85-156.
8. Ju XT, Kou CL, Christie P, Dou ZX, Zhang FS. Changes in the soil environment from excessive application of fertilizers and manures to two contrasting intensive cropping systems on the North China Plain. Environ Pollut. 2007:145(2):497-506.

9. Chien SH, Prochnow LI, Cantarella H. Recent developments of fertilizer production and use to improve nutrient efficiency and minimize environmental impacts. In: Advances in Agronomy, vol. 102. Cambridge: Academic Press; 2009. p. 267-322.

10. D-q L, Q-y T, Y-b Z, J-q Q, Li H, L-j C, S-h Y, Y-b Z, S-b P. Effect of Nitrogen Regimes on Grain Yield, Nitrogen Utilization, Radiation Use Efficiency, and Sheath Blight Disease Intensity in Super Hybrid Rice. J Integr Agric. 2012; 11(1):134-43.

11. Liu C, Zhou L, Jia J, Wang L, Si J, Li X, Pan C, Siddique KHM, Li F. Maize yield and water balance is affected by nitrogen application in a film-mulching ridge-furrow system in a semiarid region of China. Eur J Agron. 2014;52(Part B):103-11.

12. Zhang $Y$, Wang H, Lei Q, Luo J, Lindsey S, Zhang J, Zhai L, Wu S, Zhang J, Liu $X$. Optimizing the nitrogen application rate for maize and wheat based on yield and environment on the Northern China Plain. Sci Total Environ. 2018;550(7677):469.

13. Wang $X, L i Z$, Xing Y. Effects of mulching and nitrogen on soil temperature, water content, nitrate- $\mathrm{N}$ content and maize yield in the Loess Plateau of China. Agric Water Manag. 2015;161:53-64.

14. Zhu ZL, Chen D. Nitrogen fertilizer use in China - Contributions to food production, impacts on the environment and best management strategies. Nutr Cycl Agroecosyst. 2002;63(2-3):117-27.

15. Goulding $K$, Jarvis $S$, Whitmore A. Optimizing nutrient management for farm systems. Philos Trans R Soc B Biol Sci. 2008:363(1491):667-80.

16. Ning P, Fritschi FB, Li C. Temporal dynamics of post-silking nitrogen fluxes and their effects on grain yield in maize under low to high nitrogen inputs. Field Crop Res. 2017;204(Supplement C):249-59.

17. Sharma L, Bali S. A Review of methods to improve nitrogen use efficiency in agriculture. Sustainability. 2018;10(1):51.

18. Wang L, Li XG, Guan Z-H, Jia B, Turner NC, Li F-M. The effects of plastic-film mulch on the grain yield and root biomass of maize vary with cultivar in a cold semiarid environment. Field Crop Res. 2018;216:89-99.

19. Mo F, Wang J-Y, Li F-M, Nguluu SN, Ren H-X, Zhou H, Zhang J, Kariuki CW, Gicheru $P$, Kavagi $L$, et al. Yield-phenology relations and water use efficiency of maize (Zea mays L.) in ridge-furrow mulching system in semiarid east African Plateau. Sci Rep. 2017;7:3260.

20. Shang Q, Ling N, Feng X, Yang X, Wu P, Zou J, Shen Q, Guo S. Soil fertility and its significance to crop productivity and sustainability in typical agroecosystem: a summary of long-term fertilizer experiments in China. Plant Soil. 2014;381(1):13-23.

21. Gao Y, Li Y, Zhang J, Liu W, Dang Z, Cao W, Qiang Q. Effects of mulch, N fertilizer, and plant density on wheat yield, wheat nitrogen uptake, and residual soil nitrate in a dryland area of China. Nutr Cycl Agroecosyst. 2009; 85(2):109-21.

22. Wang YP, Li XG, Hai L, Siddique KHM, Gan Y, Li FM. Film fully-mulched ridge-furrow cropping affects soil biochemical properties and maize nutrient uptake in a rainfed semi-arid environment. Soil Sci Plant Nutr. 2014, 60(4):486-98.

23. Li C, Wang C, Wen X, Qin X, Liu Y, Han J, Li Y, Liao Y, Wu W. Ridge-furrow with plastic film mulching practice improves maize productivity and resource use efficiency under the wheat-maize double-cropping system in dry semi-humid areas. Field Crop Res. 2017;203(Supplement C):201-11.

24. Qin W, Hu C, Oenema O. Soil mulching significantly enhances yields and water and nitrogen use efficiencies of maize and wheat: a meta-analysis. Sci Rep. 2015:5:16210.

25. Wang X, Fan J, Xing Y, Xu G, Wang H, Deng J, Wang Y, Zhang F, Li P, Li Z. The effects of mulch and nitrogen fertilizer on the soil environment of crop plants. In: Sparks DL, editor. Advances in Agronomy, vol. 153. Cambridge: Academic Press; 2019. p. 121-73

26. Xu W, Liu C, Wang $K$, Xie R, Ming B, Wang Y, Zhang G, Liu G, Zhao R, Fan P, et al. Adjusting maize plant density to different climatic conditions across a large longitudinal distance in China. Field Crop Res. 2017;212:126-34.

27. Zhang W, Yu C, Zhang K, Zhou Y, Tan W, Zhang L, Li Z, Duan L. Plant growth regulator and its interactions with environment and genotype affect maize optimal plant density and yield. Eur J Agron. 2017;91:34-43.

28. Mhlanga B, Chauhan BS, Thierfelder $C$. Weed management in maize using crop competition: A review. Crop Prot. 2016;88:28-36. 
29. Lee E, Tollenaar M. Physiological basis of successful breeding strategies for maize grain yield. Crop Sci. 2007;47(Supplement_3):S-202-15.

30. Li J, Xie RZ, Wang KR, Ming B, Guo YQ, Zhang GQ, Li SK. Variations in maize dry matter, harvest index, and grain yield with plant density. Agron J. 2015; 107:829-34.

31. Xu C, Huang S, Tian B, Ren J, Meng Q, Wang P. Manipulating planting density and nitrogen fertilizer application to improve yield and reduce environmental impact in Chinese maize production. Front Plant Sci. 2017;8: 1234.

32. Jin L, Cui H, Li B, Zhang J, Dong S, Liu P. Effects of integrated agronomic management practices on yield and nitrogen efficiency of summer maize in North China. Field Crop Res. 2012;134:30-5.

33. Ciampitti IA, Vyn TJ. A comprehensive study of plant density consequences on nitrogen uptake dynamics of maize plants from vegetative to reproductive stages. Field Crop Res. 2011;121(1):2-18.

34. Hammad HM, Farhad W, Abbas F, Fahad S, Saeed S, Nasim W, Bakhat HF. Maize plant nitrogen uptake dynamics at limited irrigation water and nitrogen. Environ Sci Pollut Res. 2017;24(3):2549-57.

35. Chen Y, Xiao C, Wu D, Xia T, Chen Q, Chen F, Yuan L, Mi G. Effects of nitrogen application rate on grain yield and grain nitrogen concentration in two maize hybrids with contrasting nitrogen remobilization efficiency. Eur J Agron. 2015;62:79-89.

36. Hütsch BW, Schubert S: Harvest index of maize (Zea mays L.): Are there possibilities for improvement? In: Advances in Agronomy. Edited by Sparks DL, vol. 146. Cambridge: Academic Press; 2017: 37-82.

37. Mueller SM, Vyn TJ. Maize plant resilience to $\mathrm{N}$ stress and post-silking $\mathrm{N}$ capacity changes over time: A Review. Front Plant Sci. 2016;7:53.

38. Dordas C. Dry matter, nitrogen and phosphorus accumulation, partitioning and remobilization as affected by $\mathrm{N}$ and $\mathrm{P}$ fertilization and source-sink relations. Eur J Agron. 2009;30(2):129-39.

39. Dong Q, Dang T, Guo S, Hao M. Effects of mulching measures on soil moisture and $\mathrm{N}$ leaching potential in a spring maize planting system in the southern Loess Plateau. Agric Water Manag. 2019;213:803-8.

40. Seddaiu G, locola I, Farina R, Orsini R, lezzi G, Roggero PP. Long term effects of tillage practices and $\mathrm{N}$ fertilization in rainfed Mediterranean cropping systems: durum wheat, sunflower and maize grain yield. Eur J Agron. 2016; 77:166-78.

41. Sun S, Chen Z, Jiang H, Zhang L. Black film mulching and plant density influencing soil water temperature conditions and maize root growth. Vadose Zone J. 2018;17:1-12.

42. Jia Q, Sun L, Mou H, Ali S, Liu D, Zhang Y, Zhang P, Ren X, Jia Z. Effects of planting patterns and sowing densities on grain-filling, radiation use efficiency and yield of maize (Zea mays L.) in semi-arid regions. Agric Water Manag. 2018;201:287-98.

43. Guo E, Zhang J, Wang Y, Alu S, Wang R, Li D, Ha S. Assessing non-linear variation of temperature and precipitation for different growth periods of maize and their impacts on phenology in the Midwest of Jilin Province, China. Theor Appl Climatol. 2018;132(3):685-99.

44. Ding S, Su P. Effects of tree shading on maize crop within a Poplar-maize compound system in Hexi Corridor oasis, northwestern China. Agrofor Syst. 2010;80(1):117-29.

45. Xiao Q, L-x Z, Tang L, Y-f S, S-q L. Responses of crop nitrogen partitioning, translocation and soil nitrogen residue to biochar addition in a temperate dryland agricultural soil. Plant Soil. 2017:418(1):405-21.

46. Xiao Q, Zhu L-X, Zhang H-P, Li X-Y, Shen Y-F, Li S-Q. Soil amendment with biochar increases maize yields in a semi-arid region by improving soil quality and root growth. Crop Pasture Science. 2016;67(5):495-507.

47. Gu X, Ding M, Lu W, Lu D. Nitrogen topdressing at the jointing stage affects the nutrient accumulation and translocation in rainfed waxy maize. J Plant Nutr. 2019;42(6):657-72.

48. Masvaya EN, Nyamangara J, Descheemaeker K, Giller KE. Tillage, mulch and fertiliser impacts on soil nitrogen availability and maize production in semiarid Zimbabwe. Soil Tillage Res. 2017;168(Supplement C):125-32.

49. Dong Q, Dang T, Guo S, Hao M. Effect of different mulching measures on nitrate nitrogen leaching in spring maize planting system in south of Loess Plateau. Agric Water Manag. 2019;213:654-8.

50. Tian G, Kang BT, Brussaard L. Mulching effect of plant residues with chemically contrasting compositions on maize growth and nutrients accumulation. Plant Soil. 1993;153(2):179-87.
51. Barbieri P, Echarte L, Della Maggiora A, Sadras VO, Echeverria H, Andrade $\mathrm{FH}$. Maize evapotranspiration and water-use efficiency in response to row spacing. Agron J. 2012;104:939-44.

52. Bu L, Liu J, Zhu L, Luo S, Chen X, Li S, Hill RL, Zhao Y. The effects of mulching on maize growth, yield and water use in a semi-arid region. Agric Water Manag. 2013;123:71-8.

53. Zhang S, Sadras V, Chen X, Zhang F. Water use efficiency of dryland maize in the Loess Plateau of China in response to crop management. Field Crop Res. 2014;163(Supplement C):55-63.

54. Ciampitti IA, Vyn TJ. Physiological perspectives of changes over time in maize yield dependency on nitrogen uptake and associated nitrogen efficiencies: A review. Field Crop Res. 2012;133:48-67.

55. Liu Z, Gao J, Gao F, Liu P, Zhao B, Zhang J. Late harvest improves yield and nitrogen utilization efficiency of summer maize. Field Crop Res. 2019;232:88-94.

56. Wang S, Luo S, Yue S, Shen Y, Li S. Fate of $15 \mathrm{~N}$ fertilizer under different nitrogen split applications to plastic mulched maize in semiarid farmland. Nutr Cycl Agroecosyst. 2016;105(2):129-40.

57. Ma BL, Dwyer LM. Nitrogen uptake and use of two contrasting maize hybrids differing in leaf senescence. Plant Soil. 1998;199(2):283-91.

58. Hammad HM, Abbas F, Ahmad A, Farhad W, Wilkerson CJ, Hoogenboom G. Evaluation of timing and rates for nitrogen application for optimizing maize growth and development and maximizing yield. Agron J. 2018;110:565-71.

59. Rodriguez DGP, Bullock DS, Boerngen MA. The origins, implications, and consequences of yield-based nitrogen fertilizer management. Agron J. 2019; 111(2):725-35.

60. Srivastava RK, Panda RK, Chakraborty A, Halder D. Enhancing grain yield, biomass and nitrogen use efficiency of maize by varying sowing dates and nitrogen rate under rainfed and irrigated conditions. Field Crop Res. 2018; 221:339-49.

61. Liu Z, Chen Z, Ma P, Meng Y, Zhou J. Effects of tillage, mulching and N management on yield, water productivity, $N$ uptake and residual soil nitrate in a long-term wheat-summer maize cropping system. Field Crop Res. 2017; 213(Supplement C):154-64.

62. Sithole NJ, Magwaza LS, Mafongoya PL. Conservation agriculture and its impact on soil quality and maize yield: A South African perspective. Soil Tillage Res. 2016;162:55-67.

63. Quemada M, Gabriel JL. Approaches for increasing nitrogen and water use efficiency simultaneously. Global Food Security. 2016;9:29-35.

64. Zheng L, Pei J, Jin X, Schaeffer S, An T, Wang J. Impact of plastic film mulching and fertilizers on the distribution of straw-derived nitrogen in a soil-plant system based on 15N-labeling. Geoderma. 2018;317:15-22.

65. Chen P, Song C, X-m L, Zhou L, Yang H, Zhang X, Zhou Y, Du Q, Pang T, Zd $F$, et al. Yield advantage and nitrogen fate in an additive maize-soybean relay intercropping system. Sci Total Environ. 2019;657:987-99.

66. Castaldelli G, Colombani N, Soana E, Vincenzi F, Fano EA, Mastrocicco M. Reactive nitrogen losses via denitrification assessed in saturated agricultural soils. Geoderma. 2019;337:91-8.

67. Rajcan I, Swanton CJ. Understanding maize-weed competition: resource competition, light quality and the whole plant. Field Crops Res. 2001;71(2): 139-50.

68. Timlin DJ, Naidu TCM, Fleisher DH, Reddy VR. Quantitative effects of phosphorus on maize canopy photosynthesis and biomass. Crop Sci. 2017; 57:3156-69.

69. Liu T, Huang R, Cai T, Han Q, Dong S. Optimum leaf removal increases nitrogen accumulation in kernels of maize grown at high density. Sci Rep. 2017;7:39601.

70. Liu J, Zhan A, Chen H, Luo S, Bu L, Chen X, Li S. Response of nitrogen use efficiency and soil nitrate dynamics to soil mulching in dryland maize (Zea mays L.) fields. Nutr Cycl Agroecosyst. 2015;101(2):271-83.

71. Wang H, Yang Z, Yu Y, Chen S, He Z, Wang Y, Jiang L, Wang G, Yang C, Liu $B$, et al. Drought enhances nitrogen uptake and assimilation in maize roots. Agron J. 2017;109:39-46.

72. Ren B, Dong S, Zhao B, Liu P, Zhang J. Responses of nitrogen metabolism, uptake and translocation of maize to waterlogging at different growth stages. Front Plant Sci. 2017:8:1216.

73. Malhi SS, Lemke R. Tillage, crop residue and N fertilizer effects on crop yield, nutrient uptake, soil quality and nitrous oxide gas emissions in a second 4yr rotation cycle. Soil Tillage Res. 2007;96(1):269-83.

74. Wen Z, Shen J, Blackwell M, Li H, Zhao B, Yuan H. Combined applications of nitrogen and phosphorus fertilizers with manure Increase maize yield and 
nutrient uptake via stimulating root growth in a long-term experiment. Pedosphere. 2016;26(1):62-73.

75. Wang $X$, Wang N, Xing Y, Ben El Caid M. Synergetic effects of plastic mulching and nitrogen application rates on grain yield, nitrogen uptake and translocation of maize planted in the Loess Plateau of China. Sci Rep. 2018:8(1):14319.

76. Wang Y, Zhang X, Chen J, Chen A, Wang L, Guo X, Niu Y, Liu S, Mi G, Gao $Q$. Reducing basal nitrogen rate to improve maize seedling growth, water and nitrogen use efficiencies under drought stress by optimizing root morphology and distribution. Agric Water Manag. 2019;212:328-37.

77. Xie X, Shan S, Wang Y, Cao F, Chen J, Huang M, Zou Y. Dense planting with reducing nitrogen rate increased grain yield and nitrogen use efficiency in two hybrid rice varieties across two light conditions. Field Crop Res. 2019; 236:24-32.

78. Jiang R, Li X, Zhu W, Wang K, Guo S, Misselbrook T, Hatano R. Effects of the ridge mulched system on soil water and inorganic nitrogen distribution in the Loess Plateau of China. Agric Water Manag. 2018;203:277-88.

79. Meng Q, Yue S, Hou P, Cui Z, Chen X. Improving yield and nitrogen use efficiency simultaneously for maize and wheat in China: A Review. Pedosphere. 2016;26(2):137-47.

80. Meng Q, Cui Z, Yang H, Zhang F, Chen X: Establishing high-yielding maize system for sustainable intensification in China. In: Adv Agron. Edited by Sparks DL, vol. 148. Cambridge: Academic Press. 2018:85-109.

81. Yang $Q$, Liu P, Dong $S$, Zhang J, Zhao B. Effects of fertilizer type and rate on summer maize grain yield and ammonia volatilization loss in northern China. J Soils Sediments. 2019;19(5):2200-11.

82. Osterholz WR, Rinot O, Liebman M, Castellano MJ. Can mineralization of soil organic nitrogen meet maize nitrogen demand? Plant Soil. 2017;415(1):73-84.

83. Chen Q, Mu X, Chen F, Yuan L, Mi G. Dynamic change of mineral nutrient content in different plant organs during the grain filling stage in maize grown under contrasting nitrogen supply. Eur J Agron. 2016;80:137-53.

84. Maltese NE, Melchiori RJM, Maddonni GA, Ferreyra JM, Caviglia OP Nitrogen economy of early and late-sown maize crops. Field Crop Res. 2019;231:40-50

85. D-y S, Y-h L, J-w Z, Liu P, Zhao B, S-t D. Increased plant density and reduced $\mathrm{N}$ rate lead to more grain yield and higher resource utilization in summer maize. J Integr Agric. 2016;15(11):2515-28.

86. Ciampitti IA, Camberato JJ, Murrell ST, Vyn TJ. Maize nutrient accumulation and partitioning in response to plant density and nitrogen rate: I. Macronutrients. Agron J. 2013;105:783-95.

87. Fageria NK, Baligar VC. Enhancing nitrogen use efficiency in crop plants. In: Advances in Agronomy, vol. 88: Academic Press; 2005. p. 97-185.

88. Zhou B, Sun X, Wang D, Ding Z, Li C, Ma W, Zhao M. Integrated agronomic practice increases maize grain yield and nitrogen use efficiency under various soil fertility conditions. Crop J. 2019;7(4);527-38.

89. Mastrodomenico AT, Haegele JW, Seebauer JR, Below FE. Yield stability differs in commercial maize hybrids in response to changes in plant density, nitrogen fertility, and environment. Crop Sci. 2018;58:230-41.

90. Karwat H, Moreta D, Arango J, Núñez J, Rao I, Rincón Á, Rasche F, Cadisch G. Residual effect of BNI by Brachiaria humidicola pasture on nitrogen recovery and grain yield of subsequent maize. Plant Soil. 2017; 420(1):389-406

91. Gu X-B, Li Y-N, Du Y-D. Optimized nitrogen fertilizer application improves yield, water and nitrogen use efficiencies of winter rapeseed cultivated under continuous ridges with film mulching. Ind Crop Prod. 2017;109:233-40.

92. Gan Y, Siddique KHM, Turner NC, Li X-G, Niu J-Y, Yang C, Liu L, Chai Q. Ridge-furrow mulching systems - an innovative technique for boosting crop productivity in Semiarid rain-fed environments. In: Sparks DL, editor. Advances in Agronomy, vol. 118. Cambridge: Academic Press; 2013. p. 429-76.

93. Cairns JE, Sonder K, Zaidi PH, Verhulst N, Mahuku G, Babu R, Nair SK, Das B, Govaerts B, Vinayan MT, et al. Maize production in a changing climate: Impacts, adaptation, and mitigation strategies. In: Sparks DL, editor. Advances in Agronomy, vol. 114. Cambridge: Academic Press; 2012. p. 1-58.

94. Stoop WA, Uphoff N, Kassam A. A review of agricultural research issues raised by the system of rice intensification (SRI) from Madagascar: opportunities for improving farming systems for resource-poor farmers. Agric Syst. 2002;71(3):249-74.
95. Wang $X$, Wang N, Xing Y, Yun J, Zhang H. Effects of plastic mulching and basal nitrogen application depth on nitrogen use efficiency and yield in maize. Front Plant Sci. 2018;9:1446.

96. Wei S, Wang $X$, Jiang D, Dong S. Physiological and proteome studies of maize (Zea mays L.) in response to leaf removal under high plant density. BMC Plant Biol. 2018;18(1):378.

97. Deng F, Wang L, Ren W-J, Mei X-F. Enhancing nitrogen utilization and soil nitrogen balance in paddy fields by optimizing nitrogen management and using polyaspartic acid urea. Field Crop Res. 2014;169:30-8.

\section{Publisher's Note}

Springer Nature remains neutral with regard to jurisdictional claims in published maps and institutional affiliations.

\section{Ready to submit your research? Choose BMC and benefit from:}

- fast, convenient online submission

- thorough peer review by experienced researchers in your field

- rapid publication on acceptance

- support for research data, including large and complex data types

- gold Open Access which fosters wider collaboration and increased citations

- maximum visibility for your research: over $100 \mathrm{M}$ website views per year

At BMC, research is always in progress.

Learn more biomedcentral.com/submissions 